\title{
Electromagnetic Form Factors in a Collective Model of the Nucleon
}

\author{
R. Bijker ${ }^{a}$, F. Iachello ${ }^{b}$ and A. Leviatan ${ }^{c}$ \\ a Instituto de Ciencias Nucleares, U.N.A.M., \\ A.P. 70-543, 04510 México D.F., México \\ $b$ Center for Theoretical Physics, Sloane Laboratory, \\ Yale University, New Haven, CT 06520-8120, U.S.A. \\ c Racah Institute of Physics, The Hebrew University, \\ Jerusalem 91904, Israel
}

\begin{abstract}
We study the electromagnetic form factors of the nucleon in a collective model of baryons. Using the algebraic approach to hadron structure, we derive closed expressions for both elastic and transition form factors, and consequently for the helicity amplitudes that can be measured in electro- and photoproduction. Effects of spin-flavor symmetry breaking and of swelling of hadrons with increasing excitation energy are considered.
\end{abstract}

PACS numbers: 13.40.Gp, 14.20.Dh, 14.20.Gk, 11.30.Na 


\section{Introduction}

Form factors are an important ingredient in understanding the structure of hadrons. Elastic form factors of the nucleon have been measured several times [1] up to relatively large momentum transfer, $Q^{2} \approx 20(\mathrm{GeV} / \mathrm{c})^{2}$. In the absence of detailed solutions of QCD in the nonperturbative regime, they have been described by models. Traditionally, Vector Dominance Models [2] have been used to fit the data in the low $Q^{2}$ region. For $Q^{2} \gg M^{2}$, where $M$ is the nucleon mass, perturbative QCD has been used [3]. Other approaches include constituent quark models [4, QCD sum rules [5] and quark-diquark models [6]. Inelastic (transition) form factors have also been measured [7], although not as accurately as the elastic ones. A remeasurement of these form factors will form an important part of the experimental programs at various facilities, e.g. CEBAF ( $N^{*}$ collaboration) and MAMI. Extensive calculations have been carried out in the nonrelativistic and relativized quark models [8, 9, 10, 11.

In this article, we present another method which can describe simultaneously both elastic and inelastic form factors. This method is semi-phenomenological, in the sense that it assumes a certain form for the elastic form factors, and then calculates all other form factors by making use of the algebraic approach to hadron structure [12]. The main aspect of the paper is the presentation of results for form factors and helicity amplitudes in an explicit analytic form that allows one to study models of hadron structure having the same spin-flavor structure. In addition, we investigate two additional aspects of the nucleon form factors, arising from breaking of the "effective" spin-flavor symmetry in the three constituent channel, and of swelling of hadrons with increasing excitation energy. We find that, even if we attribute the entire neutron electric form factor to breaking of $S U_{s f}(6)$, this breaking still does not significantly affect other observable quantities, while the stretching of hadrons with increasing excitation energy plays a significant role. The phenomenological breaking needed to describe $G_{E}^{n}$ is much too large when compared with

QCD flavor breaking mechanisms [19], and it worsens the description of $G_{E}^{p}$. We conclude therefore, as other authors do, that meson cloud corrections play an important role in 
$G_{E}^{n}$

\section{Collective Model of Baryons}

We begin by reviewing the algebraic approach to baryon structure [12]. This approach can be used for any constituent model, but we consider in this article a collective (string-like) model with the configuration depicted in Fig. 1. The relevant degrees of freedom of this configuration are the two Jacobi coordinates

$$
\begin{aligned}
\vec{\rho} & =\frac{1}{\sqrt{2}}\left(\vec{r}_{1}-\vec{r}_{2}\right), \\
\vec{\lambda} & =\frac{1}{\sqrt{6}}\left(\vec{r}_{1}+\vec{r}_{2}-2 \vec{r}_{3}\right),
\end{aligned}
$$

where $\vec{r}_{1}, \vec{r}_{2}$ and $\vec{r}_{3}$ denote the end points of the string configuration. In the algebraic approach, the Jacobi coordinates, $\vec{\rho}$ and $\vec{\lambda}$, and their conjugate momenta, $\vec{p}_{\rho}$ and $\vec{p}_{\lambda}$, are quantized (up to a canonical transformation) with boson operators

$$
\begin{array}{ll}
b_{\rho, m}^{\dagger}=\frac{1}{\sqrt{2}}\left(\rho_{m}-i p_{\rho, m}\right), & b_{\rho, m}=\frac{1}{\sqrt{2}}\left(\rho_{m}+i p_{\rho, m}\right), \\
b_{\lambda, m}^{\dagger}=\frac{1}{\sqrt{2}}\left(\lambda_{m}-i p_{\lambda, m}\right), & b_{\lambda, m}=\frac{1}{\sqrt{2}}\left(\lambda_{m}+i p_{\lambda, m}\right),
\end{array}
$$

with $m=-1,0,1$, and an additional scalar boson, $s^{\dagger}, s$ is introduced. These operators satisfy usual boson commutation relations and operators of different type commute. Their number-conserving bilinear products generate the Lie algebra of $U(7)$ whose elements serve in the expansion of physical operators (the mass operator and transition operators). The $U(7)$ algebra enlarges the $U(6)$ algebra of the harmonic oscillator quark model [13], still describing the dynamics of two vectors. The $s$-boson does not introduce a new degree of freedom, since for a given total boson number $N$ it can always be eliminated $s \rightarrow \sqrt{N-\hat{n}_{\rho}-\hat{n}_{\lambda}}$ (Holstein-Primakoff realization of $U(7)$ ). Its introduction is just an elegant and efficient way by means of which the full dynamics of two vectors can be investigated, including those situations in which there is strong mixing of the oscillator basis (collective models). For a system of interacting bosons all states of the model space are assigned to the totally symmetric representation $[N]$ of $U(7)$. This representation 
contains all oscillator shells with $n=n_{\rho}+n_{\lambda}=0,1,2, \ldots, N$. The value of $N$ determines the size of the model space and, in view of confinement, is expected to be large. The geometric structure of baryons is thus described by the algebra of

$$
\mathcal{G}_{r} \equiv U(7)
$$

The full algebraic structure is obtained by combining this part with the internal spinflavor-color part

$$
\mathcal{G}_{i} \equiv S U_{s f}(6) \otimes S U_{c}(3)
$$

As discussed in detail in Ref. [12], the object of Fig. 1 is a top. If the three strings have equal length and equal relative angles, the top is oblate and has $D_{3 h}$ point group symmetry. The classification under $D_{3 h}$ is equivalent to the classification under permutations and parity [14]. States are characterized by $\left(v_{1}, v_{2}\right) ; K, L_{t}^{P}$, where $\left(v_{1}, v_{2}\right)$ denote the vibrations (stretching, bending); $K$ denotes the projection of the rotational angular momentum $L$ on the body-fixed symmetry axis, $P$ the parity and $t$ the symmetry type of the state under $D_{3}$ (a subgroup of $D_{3 h}$ ), or equivalently the symmetry type under $S_{3}$, the group of permutations of the three end points $\left(S_{3}\right.$ and $D_{3}$ are isomorphic). Both groups have one-dimensional symmetric and antisymmetric representations and a twodimensional representation, called $S, A, M$ for $S_{3}$ and $A_{1}, A_{2}, E$ for $D_{3}$, respectively. The notation in terms of $S_{3}$ is the one used in constituent quark models [15, 16]. The permutation symmetry of the geometric part must be the same as the permutation symmetry of the spin-flavor part in order to have total wave functions that are antisymmetric (the color part is a color singlet, i.e. antisymmetric). Therefore one can also use the dimension of the $S U_{s f}(6)$ representations to label the states: $S \leftrightarrow A_{1} \leftrightarrow 56, A \leftrightarrow A_{2} \leftrightarrow 20$, $M \leftrightarrow E \leftrightarrow 70$. In Ref. [12], a $S_{3}$-invariant mass operator was used, consisting of spatial and spin-flavor contributions, to obtain a description of the mass spectrum of nonstrange baryons with a r.m.s. deviation of $39 \mathrm{MeV}$. The nonstrange baryon resonances were identified with rotations and vibrations of the string. The corresponding wave functions, when expressed in a harmonic oscillator basis, are spread over many shells and hence are truly collective. 


\section{Electromagnetic Form Factors}

Electromagnetic form factors appear in the coupling of baryons with the electromagnetic field. In constituent models, the (point-like) constituent parts are coupled to the field [17]. The current is then reduced [9, 10] to a nonrelativistic part, a spin-orbit part and a non-additive part associated with Wigner rotations and higher order corrections. We discuss here the nonrelativistic contribution to the form factors for nonstrange baryons. Transverse, longitudinal and scalar couplings can be expressed in terms of the operators 12

$$
\begin{aligned}
\hat{U} & =\mathrm{e}^{-i k \sqrt{\frac{2}{3}} \lambda z} \\
\hat{T}_{m} & =\frac{i m_{3} k_{0}}{2}\left(\sqrt{\frac{2}{3}} \lambda_{m} \mathrm{e}^{-i k \sqrt{\frac{2}{3}} \lambda z}+\mathrm{e}^{-i k \sqrt{\frac{2}{3}} \lambda z} \sqrt{\frac{2}{3}} \lambda_{m}\right)
\end{aligned}
$$

(with $m= \pm 1,0$ ) which act on the spatial part of the baryon wave function. Here $\vec{k}=k \hat{z}$ is the photon momentum, $k_{0}$ the photon energy, and $m_{3}$ the mass of the third constituent. The form factors of interest in photo- and electroproduction as well as in elastic electron scattering are proportional to the matrix elements of these operators between initial and final states. These matrix elements can be evaluated in coordinate or momentum space as done in the nonrelativistic [17, 8] or relativized quark model [16, 9, 10, 11]. Following Ref. [12] we prefer to use an algebraic method to evaluate the matrix elements of Eq. (3.1). In order to do this, we first express the operators in Eq. (3.1) in terms of generators of the algebra of $U(7)$

$$
\begin{aligned}
\hat{U} & =\mathrm{e}^{-i k \beta \hat{D}_{\lambda, z} / X_{D}} \\
\hat{T}_{m} & =\frac{i m_{3} k_{0} \beta}{2 X_{D}}\left(\hat{D}_{\lambda, m} \mathrm{e}^{-i k \beta \hat{D}_{\lambda, z} / X_{D}}+\mathrm{e}^{-i k \beta \hat{D}_{\lambda, z} / X_{D}} \hat{D}_{\lambda, m}\right) .
\end{aligned}
$$

Here the dipole operator $\hat{D}_{\lambda, m}=\left(b_{\lambda}^{\dagger} \times s-s^{\dagger} \times \tilde{b}_{\lambda}\right)_{m}^{(1)}$, with $\tilde{b}_{\lambda, m}=(-1)^{1-m} b_{\lambda,-m}$, is a generator of $U(7)$ which transforms as a vector $\left(L^{P}=1^{-}\right)$under rotations, is even under time-reversal and has the same character under permutations as the Jacobi coordinate $\lambda_{m}$. The coefficient $X_{D}$ is a normalization factor given by the reduced matrix element $X_{D}=\left|\left\langle 1_{M}^{-}|| \hat{D}_{\lambda}|| 0_{S}^{+}\right\rangle\right|$, and $\beta$ represents the scale of the coordinate. 
The calculation of the matrix elements of these operators presents a formidable task, since it involves matrix elements of exponentiated operators. However, since the operator $\hat{D}_{\lambda, z}$ is a generator of $U(7)$, the matrix elements of $\hat{U}$ are the group elements of $U(7)$ (the generalization of the Wigner $\mathcal{D}$-functions of the rotation group) and hence can be evaluated exactly in a basis provided by the irreducible representation $[N]$ of $U(7)$. A computer program has been written to do this evaluation numerically, but in the limit of a large model space $(N \rightarrow \infty)$ the results can also be obtained in closed form. Using harmonic oscillator wave functions one recovers the familiar expressions of the nonrelativistic harmonic oscillator quark model [17, 10] (see Table VII of Ref. [12]). Explicit analytic results can also be obtained for the collective oblate top, for which partial results were presented in Tables VIII and IX of Ref. [12]. Here we present the complete results.

The evaluation of the collective form factors proceeds along the lines of the appendices of Ref. [12. We first evaluate the matrix elements of the operators in Eq. (3.2) between initial and final states which corresponds to the case in which the charge and magnetization are concentrated at the end points of the string of Fig. 1. These matrix elements are expressed in terms of spherical Bessel functions $j_{L}(k \beta)$, and are given in Table [. This table, which completes Table VIII of Ref. [12], forms the backbone from which form factors for collective models are built. We note that form factors depend only on two quantities: the scale of the coordinate $\beta$ and the quantity $R$ that measures the collectivity (see Appendix B of Ref. [12]). The latter quantity appears only in the transition form factors to the vibrational excitations of the string. In the nonrelativistic limit the resonances with $\left[20,1^{+}\right]_{(0,0) ; 0}$ and $\left[70,2^{-}\right]_{(0,0) ; 1}$ are decoupled from the nucleon ground state $\left[56,0^{+}\right]_{(0,0) ; 0}$.

Table प can be used to study form factors in collective models of the nucleon. A collective model of the nucleon is defined here as an object with the geometric shape of Fig. 1 and with a specified distribution of charge and magnetization. We consider, in particular, the model specified by the (normalized) distribution

$$
g(\beta)=\beta^{2} \mathrm{e}^{-\beta / a} / 2 a^{3},
$$


where $a$ is a scale parameter. The collective form factors are obtained by folding the matrix elements of $\hat{U}$ and $\hat{T}_{m}$ with this probability distribution

$$
\begin{aligned}
\mathcal{F}(k) & =\int \mathrm{d} \beta g(\beta)\left\langle\psi_{f}|\hat{U}| \psi_{i}\right\rangle, \\
\mathcal{G}_{m}(k) & =\int \mathrm{d} \beta g(\beta)\left\langle\psi_{f}\left|\hat{T}_{m}\right| \psi_{i}\right\rangle .
\end{aligned}
$$

Here $\psi$ denotes the spatial part of the baryon wave function. According to Table $\mathbb{\Psi}$, for large $N$ the elastic spatial matrix element of $\hat{U}$ is given by the spherical Bessel function $j_{0}(k \beta)$. The ansatz of Eq. (3.3) for the probability distribution is made to obtain the dipole form for the elastic form factor

$$
\mathcal{F}(k)=\int \mathrm{d} \beta g(\beta) j_{0}(k \beta)=1 /\left(1+k^{2} a^{2}\right)^{2}
$$

Closed expressions for selected collective transition form factors of the distributed string are given in Table [1, which completes Table IX of Ref. [12]. It is instructive to study both the small and large $k$ dependence of the form factors. This dependence is given in Table [II]. For small values of $k$ the transition form factors $\mathcal{F}(k)$ behave as $\sim k^{L}$ for rotational excitations with $v=v_{1}+v_{2}=0$ and orbital angular momentum $L$, and as $\sim k^{2}$ for vibrational excitations with $v=1$ and $L=0$. More interestingly, for large values of $k$, all form factors drop as powers of $k$. This property is well-known experimentally and is in contrast with harmonic oscillator quark models in which all form factors fall off exponentially [17, 8, 10]. The elastic form factor $\mathcal{F}(k)$ drops as $k^{-4}$ (by construction), whereas the transition form factors for all rotational excitations with $v=v_{1}+v_{2}=0$ drop as $k^{-3}$. For vibrational excitations with $v=1$ and $L=0$, it drops as $k^{-4}$. The form factors $\mathcal{G}_{m}(k)$ drop as the derivatives of $\mathcal{F}(k)$.

\section{Experimental Observables}

The form factors of Section 3 can be used to calculate quantities which can be measured. We begin with the elastic electric and magnetic form factors of the nucleon. The elastic 
collective form factors are given by

$$
\begin{aligned}
& G_{E}^{N}=3 \int \mathrm{d} \beta g(\beta)\left\langle\Psi ; M_{J}=1 / 2\left|e_{3} \hat{U}\right| \Psi ; M_{J}=1 / 2\right\rangle, \\
& G_{M}^{N}=3 \int \mathrm{d} \beta g(\beta)\left\langle\Psi ; M_{J}=1 / 2\left|\mu_{3} e_{3} \sigma_{3, z} \hat{U}\right| \Psi ; M_{J}=1 / 2\right\rangle,
\end{aligned}
$$

where $\Psi$ denotes the nucleon wave function ${ }^{2} 8_{1 / 2}^{N}\left[56,0^{+}\right]_{(0,0) ; 0}$ with $N=p, n$. Further $e_{3}$, $\mu_{3}=e g_{3} / 2 m_{3}, m_{3}, g_{3}, s_{3}=\sigma_{3} / 2$ are the charge (in units of $e: e_{u}=2 / 3, e_{d}=-1 / 3$ ), scale magnetic moment, mass, $g$-factor and spin, respectively, of the third constituent. Using the results of Table [1] we obtain

$$
\begin{aligned}
G_{E}^{p} & =\frac{1}{\left(1+k^{2} a^{2}\right)^{2}}, \\
G_{E}^{n} & =0,
\end{aligned}
$$

for the charge form factors. The corresponding proton charge radius is found to be

$$
\left\langle r^{2}\right\rangle_{E}^{p}=12 a^{2}
$$

Similarly, we obtain for the magnetic form factors

$$
\begin{aligned}
G_{M}^{p} & =\frac{\mu}{\left(1+k^{2} a^{2}\right)^{2}} \\
G_{M}^{n} & =\frac{-2 \mu}{3\left(1+k^{2} a^{2}\right)^{2}} .
\end{aligned}
$$

The corresponding magnetic moments are

$$
\begin{aligned}
& \mu_{p}=\mu, \\
& \mu_{n}=-2 \mu / 3,
\end{aligned}
$$

respectively. Here we have assumed that the mass and the $g$-factor of the up $(u)$ and down $(d)$ constituents are identical, $m_{u}=m_{d}=m_{q}$ and $g_{u}=g_{d}=g$. Hence $\mu_{u}=\mu_{d}=\mu$ in Eq. (4.4) is given by $\mu=e g / 2 m_{q}$. The proton and neutron magnetic radii are identical to the proton charge radius of Eq. (4.3). The form factors in Eqs. (4.2) and (4.4) satisfy $G_{M}^{p}=\mu G_{E}^{p}$ and obey the $S U_{s f}(6)$ relations $G_{E}^{n}=0$ and $G_{M}^{n} / G_{M}^{p}=-2 / 3$. 
Other (observable) quantities of interest are the helicity amplitudes in photo- and electroproduction. The transverse helicity amplitudes between the initial (ground) state of the nucleon and the final (excited) state of a baryon resonance are expressed as 12

$$
A_{\nu}^{N}=6 \sqrt{\frac{\pi}{k_{0}}}[k\langle L, 0 ; S, \nu \mid J, \nu\rangle \mathcal{B}-\langle L, 1 ; S, \nu-1 \mid J, \nu\rangle \mathcal{A}],
$$

where $\nu=1 / 2,3 / 2$ indicates the helicity. The orbit- and spin-flip amplitudes $(\mathcal{A}$ and $\mathcal{B}$, respectively) are given by

$$
\begin{aligned}
& \mathcal{B}=\int \mathrm{d} \beta g(\beta)\left\langle\Psi_{f} ; M_{J}=\nu\left|\mu_{3} e_{3} s_{3,+} \hat{U}\right| \Psi_{i} ; M_{J}^{\prime}=\nu-1\right\rangle, \\
& \mathcal{A}=\int \mathrm{d} \beta g(\beta)\left\langle\Psi_{f} ; M_{J}=\nu\left|\mu_{3} e_{3} \hat{T}_{+} / g_{3}\right| \Psi_{i} ; M_{J}^{\prime}=\nu-1\right\rangle .
\end{aligned}
$$

Here $\left|\Psi_{i}\right\rangle$ denotes the (space-spin-flavor) wave function of the initial nucleon with ${ }^{2} 8_{1 / 2}^{N}\left[56,0^{+}\right]_{(0,0) ; 0}$ and $N=p, n$, and, similarly, $\left|\Psi_{f}\right\rangle$ that of the final baryon resonance. The helicity amplitudes extracted from experiment include the sign of the subsequent strong decay into the $\pi N$ channel and an extra conventional sign $-(+)$ for nucleon (delta) resonances [11]. Therefore, to compare with the experimental results, we multiply the helicity amplitudes of Eq. (4.6) with a coefficient $\zeta=-\operatorname{sign}\left(N^{*} \rightarrow N \pi\right.$ ) for nucleon resonances and $\zeta=+\operatorname{sign}\left(\Delta^{*} \rightarrow N \pi\right)$ for delta resonances 8 . Although the extraction of this sign and of the resultant helicity amplitudes is model dependent, we shall conform in this article with standard practice and extract the sign from a calculation of strong decays in a simple model, in which it is assumed that the pion is emitted from a single constituent and which uses the same collective wave functions [18. The values of $\zeta$, corresponding to the lowest nucleon and delta resonances, are shown in Tables $\mathrm{IV}$ and $\mathrm{\nabla}$.

When comparing with the experimental data one must still choose a reference frame which determines the relation between the three-momentum $k^{2}$ and the four-momentum $Q^{2}=k^{2}-k_{0}^{2}$. It is convenient to choose the equal momentum or Breit frame where

$$
k^{2}=Q^{2}+\frac{\left(W^{2}-M^{2}\right)^{2}}{2\left(M^{2}+W^{2}\right)+Q^{2}} .
$$

Here $M$ is the nucleon mass, $W$ is the mass of the resonance, and $-Q^{2}=k_{0}^{2}-k^{2}$ can be interpreted as the mass squared of the virtual photon. For elastic scattering we have $k^{2}=Q^{2}$. 
If we assume $m_{u}=m_{d}=m_{q}$ and $g_{u}=g_{d}=g$ then, just as in the case of the nucleon electric and magnetic form factors, $\mu_{u}=\mu_{d}=\mu$ in Eq. (4.7). In general, the $\mathcal{B}$ and $\mathcal{A}$ amplitudes of Eq. (4.7) are proportional to the collective form factors $\mathcal{F}$ and $\mathcal{G}_{+}$of Eq. (3.4), respectively. Explicit expressions for the helicity amplitudes of Eq. (4.6) can be obtained by combining the corresponding entries of Table $\square$ with the appropriate spin-flavor matrix elements [12]. Some of these are given in Tables $\mathbb{I V}$ and $\mathrm{V}$.

\section{$5 \quad$ Breaking of Spin-Flavor Symmetry}

In the preceeding sections we have assumed $S U_{s f}(6)$ spin-flavor symmetry. This leads to $G_{E}^{n}=0$ and $G_{M}^{n} / G_{M}^{p}=-2 / 3$ for all values of the momentum transfer, which is not obeyed by the experimental data. Within a truncated three-constituents configuration space, in order to have a nonvanishing neutron electric form factor, as experimentally observed, one must break $S U_{s f}(6)$ [20]. This breaking can be achieved in various ways, e.g. by including in the mass operator a hyperfine interaction [21], or by breaking the $D_{3}$ spatial symmetry allowing for a quark-diquark structure [22] and flavor-dependent mass terms. Within the model discussed here (an effective model with three constituent parts), we study the breaking of the $S U_{s f}(6)$ symmetry by assuming a flavor-dependent distribution of the charge and the magnetization along the strings of Fig. 1,

$$
\begin{aligned}
& g_{u}(\beta)=\beta^{2} \mathrm{e}^{-\beta / a_{u}} / 2 a_{u}^{3}, \\
& g_{d}(\beta)=\beta^{2} \mathrm{e}^{-\beta / a_{d}} / 2 a_{d}^{3} .
\end{aligned}
$$

With this dependence, the electric nucleon form factors become

$$
\begin{aligned}
G_{E}^{p} & =\frac{2 e_{u}}{\left(1+k^{2} a_{u}^{2}\right)^{2}}+\frac{e_{d}}{\left(1+k^{2} a_{d}^{2}\right)^{2}}, \\
G_{E}^{n} & =\frac{2 e_{d}}{\left(1+k^{2} a_{d}^{2}\right)^{2}}+\frac{e_{u}}{\left(1+k^{2} a_{u}^{2}\right)^{2}} .
\end{aligned}
$$

The corresponding proton and neutron charge radii are given by

$$
\begin{aligned}
\left\langle r^{2}\right\rangle_{E}^{p} & =12\left(2 e_{u} a_{u}^{2}+e_{d} a_{d}^{2}\right), \\
\left\langle r^{2}\right\rangle_{E}^{n} & =12\left(2 e_{d} a_{d}^{2}+e_{u} a_{u}^{2}\right) .
\end{aligned}
$$


In the limit $k \rightarrow \infty$, the electric form factors behave as

$$
\begin{aligned}
& G_{E}^{p} \rightarrow \frac{1}{k^{4}}\left[\frac{2 e_{u}}{a_{u}^{4}}+\frac{e_{d}}{a_{d}^{4}}\right], \\
& G_{E}^{n} \rightarrow \frac{1}{k^{4}}\left[\frac{2 e_{d}}{a_{d}^{4}}+\frac{e_{u}}{a_{u}^{4}}\right] .
\end{aligned}
$$

If the length of the string in Fig. 1 is slightly different for $u$ and $d$, so is their mass and thus in principle, their magnetic moment. Applying the same procedure to the magnetic form factors gives

$$
\begin{aligned}
G_{M}^{p} & =\frac{4 \mu_{u} e_{u}}{3\left(1+k^{2} a_{u}^{2}\right)^{2}}-\frac{\mu_{d} e_{d}}{3\left(1+k^{2} a_{d}^{2}\right)^{2}} \\
G_{M}^{n} & =\frac{4 \mu_{d} e_{d}}{3\left(1+k^{2} a_{d}^{2}\right)^{2}}-\frac{\mu_{u} e_{u}}{3\left(1+k^{2} a_{u}^{2}\right)^{2}} .
\end{aligned}
$$

where $\mu_{u} e_{u}$ and $\mu_{d} e_{d}$ are the magnetic moments of the $u$ and $d$ constituents. The proton and neutron magnetic moments are now

$$
\begin{aligned}
& \mu_{p}=\left(4 \mu_{u} e_{u}-\mu_{d} e_{d}\right) / 3, \\
& \mu_{n}=\left(4 \mu_{d} e_{d}-\mu_{u} e_{u}\right) / 3,
\end{aligned}
$$

and the proton and neutron magnetic radii are given by

$$
\begin{aligned}
\left\langle r^{2}\right\rangle_{M}^{p} & =12\left(4 \mu_{u} e_{u} a_{u}^{2}-\mu_{d} e_{d} a_{d}^{2}\right) /\left(4 \mu_{u} e_{u}-\mu_{d} e_{d}\right) \\
\left\langle r^{2}\right\rangle_{M}^{n} & =12\left(4 \mu_{d} e_{d} a_{d}^{2}-\mu_{u} e_{u} a_{u}^{2}\right) /\left(4 \mu_{d} e_{d}-\mu_{u} e_{u}\right)
\end{aligned}
$$

The asymptotic limit $\left(k^{2} \rightarrow \infty\right)$ of the magnetic form factors is

$$
\begin{aligned}
& G_{M}^{p} \rightarrow \frac{1}{k^{4}}\left[\frac{4 \mu_{u} e_{u}}{3 a_{u}^{4}}-\frac{\mu_{d} e_{d}}{3 a_{d}^{4}}\right] \\
& G_{M}^{n} \rightarrow \frac{1}{k^{4}}\left[\frac{4 \mu_{d} e_{d}}{3 a_{d}^{4}}-\frac{\mu_{u} e_{u}}{3 a_{u}^{4}}\right]
\end{aligned}
$$

We note at this stage that if the masses of the up and down constituents are slightly different, $S_{3}\left(D_{3}\right)$ symmetry is also broken in the wave functions and spectrum, causing a splitting of the degenerate rotations and vibrations. This effect will be analyzed in detail when studying strange baryons where it is much larger due to the large difference 
in the mass of the strange constituent relative to that of the up and down constituents. (We also note that our main interest is to present results for observable quantities due to spin-flavor breaking in a truncated space, independently from its magnitude. Different QCD spin flavor mechanisms give different values for the effective masses, $m_{d}$ and $m_{u}$, magnetic moments $\mu_{d}$ and $\mu_{u}$, and sizes $a_{u}$ and $a_{d}$, both with $a_{u}<a_{d}$ and $a_{u}>a_{d}$ [19.)

The breaking of spin-flavor symmetry has also influence on the helicity amplitudes. Inserting the appropriate spin-flavor coefficients in Eq. (4.7) one obtains the results for the orbit- and spin-flip amplitudes, $\mathcal{A}$ and $\mathcal{B}$, given in Tables VI and VII. The helicity amplitudes of Eq. (4.6) are now given in terms of the flavor-dependent collective form factors $\mathcal{F}_{u}(k), \mathcal{G}_{u,+}(k)$ and $\mathcal{F}_{d}(k), \mathcal{G}_{d,+}(k)$, which depend on the size parameters, $a_{u}$ and $a_{d}$, respectively. Explicit expressions for the various helicity amplitudes are available on request.

Table VI shows that two sets of helicity amplitudes which were previously zero due to spin-flavor symmetry, are nonvanishing in the presence of flavor-dependent distributions: (i) the Moorehouse selection rule for the proton helicity amplitudes for the ${ }^{4} 8_{J}[70, L]$ resonances is broken, and (ii) the neutron helicity-3/2 amplitudes for the ${ }^{2} 8_{J}[56, L]$ resonances are nonvanishing.

\section{$6 \quad$ Stretchable Strings}

In a string-like model of hadrons one expects on the basis of QCD [23, 24] that strings will elongate (hadrons swell) as their energy increases. This effect can be easily included in the present analysis by making the scale parameters of the strings energy dependent. In order to study the swelling of hadrons with increasing excitation energy, we use here the simple ansatz

$$
a=a_{0}\left(1+\xi \frac{W-M}{M}\right)
$$

where $M$ is the nucleon mass and $W$ the resonance mass. This ansatz introduces a new parameter, the stretchability of the string, $\xi$. The arguments of Ref. [23] and the analysis 
of the experimental mass spectrum (Regge trajectories) suggest $\xi \approx 1$. Spin-flavor $S U_{s f}(6)$ symmetry breaking may also effect the value of $\xi$, but this is likely to be a higher order effect. Hence we parameterize the breaking as

$$
\begin{aligned}
& a_{u}=a_{u, 0}\left(1+\xi \frac{W-M}{M}\right), \\
& a_{d}=a_{d, 0}\left(1+\xi \frac{W-M}{M}\right) .
\end{aligned}
$$

i.e. we assume the stretchability to be flavor independent.

\section{Analysis of Experimental Data}

\subsection{Spin-Flavor Breaking}

In this section we investigate the effect of the flavor dependence on the elastic and transition form factors of nonstrange baryons. We begin by discussing the determination of the parameters. For all cases we take $g_{u}=g_{d}=1$. For the calculations in which the $S U_{s f}(6)$ symmetry is satisfied $\left(\mu_{u}=\mu_{d}=\mu\right.$ and $\left.a_{u}=a_{d}=a\right)$, we determine the scale magnetic moment $\mu$ from the proton magnetic moment $\mu=\mu_{p}=2.793 \mu_{N}$, which corresponds to a constituent mass of $m_{u}=m_{d}=0.336 \mathrm{GeV}$. Since the values of the helicity amplitudes $A_{\nu}^{N}$ are usually given in $\mathrm{GeV}^{-1 / 2}$, we express the scale magnetic moment appearing in Eq. (4.7) in units of $\mu=0.127 \mathrm{GeV}^{-1}(\hbar=c=1)$. In 12 the scale parameter $a$ was determined from the proton charge radius (see Eq. (4.3)). Here we prefer to use a simultaneous fit to the proton and neutron charge radii [25, 26], to the proton electric and magnetic form factors up to $Q^{2}=5(\mathrm{GeV} / \mathrm{c})^{2}$ [27, and to the neutron electric [28] and magnetic form factors [29, 30, 31] up to $Q^{2}=4(\mathrm{GeV} / \mathrm{c})^{2}$. As a result we find $a=0.232$ fm.

In order to study the sensitivity of the form factors (elastic and transition) to breaking of $S U_{s f}(6)$ symmetry, we assume that the constituent masses $m_{u}$ and $m_{d}$ are determined from the magnetic moments with quark $g$-factors $g_{u}=g_{d}=1$. Using $\mu_{u}=2.777 \mu_{N}$ and $\mu_{d}=2.915 \mu_{N}$, we find $m_{u}=0.338 \mathrm{GeV}$ and $m_{d}=0.322 \mathrm{GeV}$, respectively. The scale 
parameters $a_{u}$ and $a_{d}$ are determined from a simultaneous fit to the proton and neutron charge radii and the proton and neutron electric and magnetic form factors, $a_{u}=0.230$ $\mathrm{fm}$ and $a_{d}=0.257 \mathrm{fm}$. We note that the magnitude of the breaking both in the effective masses and scales so determined is too large when compared with estimates based on the $m_{u}-m_{d}$ mass difference of the "current" quarks and on QCD perturbation estimates with $\alpha_{s}=0.5$ 19. The necessity to use $a_{u} \neq a_{d}$ in the present model should be interpreted as a consequence of the truncation of configuration space to the pure three-constituents states. Our purpose, however, is to understand what happens to the form factors when one breaks $S U_{s f}(6)$ in the truncated space. Since the $a_{u}$ and $a_{d}$ are effective quantities that incorporate all complexities of the non-three-constituents configurations, they may have significant final-state dependence (which is ignored in the present study).

We first discuss the elastic form factors. Figs. 2 and 3 show the electric form factors of the neutron and the proton divided by the dipole form, $F_{D}=1 /\left(1+Q^{2} / 0.71\right)^{2}$. The division by $F_{D}$ emphasizes the effect of the breaking of spin-flavor symmetry. Figs. 4 and 5 show the results for the neutron and proton magnetic form factors, respectively. We see that while the breaking of spin-flavor symmetry can account for the non-zero value of $G_{E}^{n}$ and gives a good description of the data, it worsens the fit to the proton electric and neutron magnetic form factors. This implies that, in addition to not being of the right order of magnitude when compared with QCD estimates, the simple mechanism for spin-flavor breaking discussed in Sect. 5 does not produce the right phenomenology and other contributions, such as polarization of the neutron into $p+\pi^{-}$, play an important role in the neutron electric form factor [19]. (A coupling to the meson cloud through $\rho$, $\omega$ and $\phi$ mesons is indeed expected to contribute in this range of $Q^{2}$, see Fig. 1 of Ref. [2].) This conclusion (i.e. worsening the proton form factors) applies also to the other mechanisms of spin-flavor symmetry breaking mentioned above, such as that induced by a hyperfine interaction [21] which gives $a_{u}<a_{d}$ ('moves the up quark to the center and the down quark to the periphery') although it was not discussed in Ref. [21]. This pattern is a consequence of the fact that within the framework of constituent models $G_{E}^{p}, G_{E}^{n}, G_{M}^{p}$ and $G_{M}^{n}$ are intertwined. 
We note in passing that spin-flavor breaking also alters the ratio of the magnetic form factors $G_{M}^{n} / G_{M}^{p}$. From Eq. (5.8) we find that for $k^{2} \rightarrow \infty$ this ratio approaches $G_{M}^{n} / G_{M}^{p} \rightarrow\left(4 \mu_{d} e_{d} a_{u}^{4}-\mu_{u} e_{u} a_{d}^{4}\right) /\left(4 \mu_{u} e_{u} a_{d}^{4}-\mu_{d} e_{d} a_{u}^{4}\right)$. With the values of $a_{u}, a_{d}$ and of $\mu_{u}, \mu_{d}$ given above, we calculate this ratio to be -0.541 . On the basis of perturbative QCD the ratio is expected to approach $-1 / 2+\mathcal{O}\left(\ln Q^{2}\right)$ for large values of $Q^{2}$ [9]. With harmonic oscillator form factors this ratio approaches $-1 / 4$. Without the breaking of the spin-flavor symmetry this ratio is $-2 / 3$ independent of $Q^{2}$ for both the collective and the harmonic oscillator case. The breaking of spin-flavor symmetry brings the value of the ratio for $Q^{2} \rightarrow \infty$ closer to the p-QCD value. From Fig. 6 we can see that the experimental situation does not show any indication that the perturbative regime has been reached, at least up to $Q^{2} \leq 3(\mathrm{GeV} / \mathrm{c})^{2}$.

Next we discuss the transverse helicity amplitudes $A_{1 / 2}$ and $A_{3 / 2}$. The results of the calculations with and without spin-flavor breaking are shown in Figs. 7-11 for nucleon resonances and in Figs. 12-15 for delta resonances. From these figures it is seen that the effect is rather small. Only in those cases in which the amplitude with $S U_{s f}(6)$ symmetry is zero, the effect is of some relevance. Such is the case for the neutron amplitude $A_{3 / 2}^{n}$ of the $N(1680) F_{15}$ resonance shown in Fig. 11, and for the proton amplitudes of the $N(1650) S_{11}$ (see Fig. 9), $N(1675) D_{15}$ and $N(1700) D_{13}$ resonances, which all belong to the ${ }^{4} 8_{J}\left[70, L=1^{-}\right]$multiplet. The small effect of the spin-flavor symmetry breaking is emphasized in Figs. 16-18 where the helicity asymmetries

$$
A=\frac{A_{1 / 2}^{2}-A_{3 / 2}^{2}}{A_{1 / 2}^{2}+A_{3 / 2}^{2}}
$$

are plotted versus $Q^{2}$. The conclusion that one can draw from this analysis is that, for all purposes, except the electric form factor of the neutron, the breaking of spin-flavor symmetry according to the mechanism of Sect. 5 is of little importance. As an additional comment, we note that in Figs. 8 and 9 we have shown only the amplitudes with no mixing, $\theta=0^{\circ}$ (see Eq. (10.3) of Ref. [12]), since our purpose is that of displaying the effects of spin-flavor breaking induced by $a_{u}<a_{d}$. (The mixing between the two $S_{11}$ states may be effected by meson cloud corrections, specifically, $N-\eta$ contributions.) 
The helicity amplitudes shown in Figs. 7-15 all describe rotational excitations in the collective model. It is of interest to comment briefly on vibrational excitations. As one

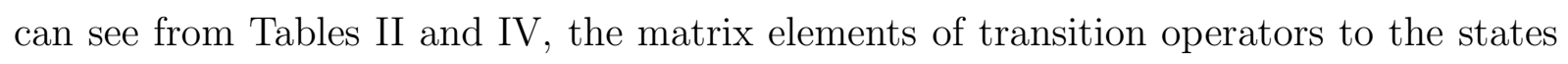
$\left[56,0^{+}\right]_{(1,0) ; 0}$ and $\left[70,0^{+}\right]_{(0,1) ; 0}$ vanish in the large $N$ limit of the collective model (and $R^{2} \neq 0$ ). Nevertheless, it is instructive to study these matrix elements for finite $N$ (but large) and $R^{2} \neq 0,1$. Denoting by $\chi=\left(1-R^{2}\right) / R \sqrt{N}$ the strength of the coupling, we show in Fig. 19 the corresponding transverse helicity amplitude for $N(1440) P_{11}$ (the Roper resonance). We note that the calculated amplitude has the opposite sign of the experimental amplitude (just as in [11, as well as in the harmonic oscillator limit of the algebraic model [12]). However, the behavior of the amplitude with $Q^{2}$ is particular enough to be able to say something concerning the nature of the Roper resonance once more accurate data will be available.

\section{2 $\quad$ Stretching}

In this section we analyze what happens to the helicity amplitudes with the stretching mechanism of Sect. 6. Figs. 20-22 show the effect of stretching on the helicity amplitudes for $\Delta(1232) P_{33}, N(1520) D_{13}$ and $N(1680) F_{15}$. It is seen that the effect of stretching, especially if one takes the value $\xi \approx 1$ suggested by the arguments of [23] and the Regge behavior of nucleon resonances (see e.g. Fig. 5 of [12]), is rather large. In particular, the data for $N(1520) D_{13}$ and $N(1680) F_{15}$ show a clear indication that the form factors are dropping faster than expected on the basis of the dipole form. (Of course for the elastic form actors there is no stretching.) We suggest that future data at CEBAF and MAMI be used to analyze the effects of stretching on the helicity amplitudes.

\section{Summary and conclusions}

In this article, we have exploited the algebraic approach to baryon structure introduced in [12] to analyze simultaneously elastic form factors and helicity amplitudes in photo- and electroproduction. The use of algebraic methods allows us to study different situations, 
such as the harmonic oscillator quark model and the collective model, within the same framework. The logic of the method is that, by starting from the charge and magnetization distribution of the ground state (assuming a dipole form to the elastic form factor of the nucleon), one can obtain the transition form factors to the excited states. In the 'collective' model, this procedure yields a power dependence of all form factors (elastic and inelastic) on $Q^{2}$. We have analyzed two aspects of hadronic structure: (i) the breaking of $S U_{s f}(6)$ symmetry, and (ii) the stretching of hadrons with increasing excitation energy. We find that, whereas the breaking of the spin-flavor symmetry hardly effects the helicity amplitudes, the stretching of hadrons does have a noticeable influence.

The disagreement between experimental and theoretical elastic form factors and helicity amplitudes in the low- $Q^{2}$ region $0 \leq Q^{2} \leq 1(\mathrm{GeV} / \mathrm{c})^{2}$, requires further investigation. We think that this disagreement is due to coupling of the photon to the meson cloud, (i.e. configurations of the type $\left.q^{3}-q \bar{q}\right)$. In the case of the elastic form factors, these effects were in part analyzed in vector dominance models [2] by writing the amplitude as the sum of two terms. We think that this analysis (which was done before the advent of quark model calculations) should be repeated by using for the 'intrinsic' part the constituent form factors discussed in this article. Coupling of the photon to $\rho$ (isovector), $\omega$ and $\phi$ (isoscalar) vector mesons can produce a non-zero neutron form factor which describes the data without worsening the proton form factor description. For the helicity amplitudes, the effects could either be calculated directly [35, or be parametrized by meson (not necessarily vector) dominance models. We note however that in either case, since configurations of the type $q^{3}-q \bar{q}$ have much larger spatial extent than $q^{3}$, these effects are expected to drop faster with momentum transfer $Q^{2}$ than the constituent form factors. Also, since meson exchange corrections contribute differently to different channels, this effect will be state dependent.

Another aspect that requires further investigation is the contribution of the spin-orbit and non-additive part in the transition operators. Since the algebraic formulation is now in place, these effects can be investigated. The corresponding results will be reported elsewhere. 


\section{Acknowledgements}

This work is supported in part by CONACyT, México under project 400340-5-3401E, DGAPA-UNAM under project IN105194 (R.B.), by D.O.E. Grant DE-FG02-91ER40608 (F.I.) and by grant No. 94-00059 from the United States-Israel Binational Science Foundation (BSF), Jerusalem, Israel (A.L.). 


\section{References}

[1] For recent measurements, see P.E. Bosted et al, in "Baryons '92", M. Gai, ed., World Scientific, Singapore (1993), p. 268.

[2] F. Iachello, A.D. Jackson and A. Lande, Phys. Lett. B43, 191 (1973).

G. Höhler, E. Pietarinen, I. Sabba-Stefanescu, F. Borkowski, G.G. Simon, V.H. Walther and R.D. Wendling, Nucl. Phys. B114, 505 (1976).

[3] G. P. Lepage and S. J. Brodsky, Phys. Rev. Lett. 43, 545 (1977); 43, 1625 (1977).

[4] P. L. Chung and F. Coester, Phys. Rev. D44, 229 (1991).

[5] A.V. Radyushkin, Acta Phys. Pol. B15, 403 (1984).

[6] P. Krole, M. Schürmann, and W. Schweizer, Z. Phys. 338, 333 (1991).

[7] See, for example, V.D. Burkert, in "Baryons '92", M. Gai, ed., World Scientific, Singapore (1993), p. 38.

[8] R. Koniuk and N. Isgur, Phys. Rev. D21, 1868 (1980).

[9] M. Warns, H. Schröder, W. Pfeil and H. Rollnik, Z. Phys. C45, 627 (1990);

M. Warns, W. Pfeil and H. Rollnik, Phys. Rev. D42, 2215 (1990).

[10] F.E. Close and Z. Li, Phys. Rev. D42, 2194 (1990);

Z. Li and F.E. Close, Phys. Rev. D42, 2207 (1990).

[11] S. Capstick, Phys. Rev. D46, 2864 (1992);

S. Capstick and B.D. Keister, Phys. Rev. D51, 3598 (1995).

[12] R. Bijker, F. Iachello and A. Leviatan, Ann. Phys. (N.Y.) 236, 69 (1994).

[13] K.C. Bowler, P.J. Corvi, A.J.G. Hey, P.D. Jarvis and R.C. King, Phys. Rev. D24, 197 (1981); A.J.G. Hey and R.L. Kelly, Phys. Rep. 96, 71 (1983). 
[14] P.R. Bunker, "Molecular Symmetry and Spectroscopy", Academic Press, New York (1979).

[15] N. Isgur and G. Karl, Phys. Rev. D18, 4187 (1978); ibid. D19, 2653 (1979); ibid. D20, 1191 (1979); K.-T. Chao, N. Isgur and G. Karl, Phys. Rev. D23, 155 (1981).

[16] S. Capstick and N. Isgur, Phys. Rev. D34, 2809 (1986).

[17] L.A. Copley, G. Karl and E. Obryk, Phys. Lett. B29, 117 (1969);

L.A. Copley, G. Karl and E. Obryk, Nucl. Phys. B13, 303 (1969).

[18] R. Bijker, F. Iachello and A. Leviatan, in preparation.

[19] F.E. Close and R.R. Hogan, Nucl. Phys. B185, 333 (1981);

O.V. Mawell and V. Vento. Nucl. Phys. A407, 366 (1983);

S. Theberge, A.W. Thomas and G.A. Miller, Phys. Rev. D22, 2838 (1980).

[20] J.L. Friar, Part. and Nucl. 4, 153 (1972);

R.D. Carlitz, S.D. Ellis and R. Savit, Phys. Lett. B68, 443 (1977).

[21] N. Isgur, G. Karl and D.W.L. Sprung, Phys. Rev. D23, 163 (1981).

[22] Y. Tzeng and S.S. Hsiao, Il Nuovo Cimento 106, 573 (1993).

[23] K. Johnson and C.B. Thorn, Phys. Rev. D13, 1934 (1974).

[24] I. Bars and H.J. Hanson, Phys. Rev. D13, 1744 (1974).

[25] G.G. Simon, Ch. Schmitt, F. Borkowski and V.H. Walther, Nucl. Phys. A333, 381 (1980).

[26] V.E. Krohn and G.R. Ringo, Phys. Rev. D8, 1305 (1973);

L. Koester, W. Nistler and W. Waschkowski, Phys. Rev. Lett. 36, 1021 (1976);

S. Kopecky, P. Riehs, J.A. Harvey and N.W. Hill, Phys. Rev. Lett. 74, 2427 (1995).

[27] R.C. Walker et al., Phys. Rev. D49 5671 (1994). 
[28] S. Platchkov et al., Nucl. Phys. A510, 740 (1990).

[29] A. Lung et al., Phys. Rev. Lett. 70, 718 (1993).

[30] E.E.W. Bruins et al., Phys. Rev. Lett. 75, 21 (1995).

[31] W. Bartel, F.W. Büsser, W.R. Dix, R. Felst, D. Harms. H. Krehbiel, P.E. Kuhlmann, J. McElroy, J. Meyer and G. Weber, Nucl. Phys. B58, 429 (1973).

[32] L. Andivahis et al., Phys. Rev. D50, 5491 (1994).

[33] Particle Data Group, Phys. Rev. D50, 1173 (1994).

[34] V.D. Burkert, in "Excited Baryons '88", G. Adams, N.C. Mukhopadhyay and P. Stoler eds., World Scientific (1989) p. 122;

V.D. Burkert, in "Perspectives in the Structure of Hadronic Systems", M.N. Harakeh and J.H. Hoch ed., Plenum Press, (1994), p. 101.

[35] T.-S. H. Lee, in "Baryons '92", M. Gai, ed., World Scientific, Singapore (1993), p. 99. 
Table I: Matrix elements of the transition operators of Eq. (3.2) in the large $N$ limit. The final states are labeled by $\left[\operatorname{dim}, L^{P}\right]_{\left(v_{1}, v_{2}\right) ; K}$, where $\operatorname{dim}$ denotes the dimension of the $S U_{s f}(6)$ representation. The initial state is $\left[56,0^{+}\right]_{(0,0) ; 0}$.

Final state $\quad\left\langle\psi_{f}|\hat{U}| \psi_{i}\right\rangle \quad\left\langle\psi_{f}\left|\hat{T}_{0}\right| \psi_{i}\right\rangle / m_{3} k_{0} \beta \quad\left\langle\psi_{f}\left|\hat{T}_{ \pm}\right| \psi_{i}\right\rangle / m_{3} k_{0} \beta$

$\left[56,0^{+}\right]_{(0,0) ; 0} \quad j_{0}(k \beta) \quad j_{1}(k \beta) \quad 0$

$\left[20,1^{+}\right]_{(0,0) ; 0}$

0

0

0

$\left[70,1^{-}\right]_{(0,0) ; 1} \quad-i \sqrt{3} j_{1}(k \beta)$

$i \frac{1}{\sqrt{3}}\left[j_{0}(k \beta)-2 j_{2}(k \beta)\right]$

$\mp i \sqrt{\frac{2}{3}}\left[j_{0}(k \beta)+j_{2}(k \beta)\right]$

$\left[56,2^{+}\right]_{(0,0) ; 0}$

$\frac{1}{2} \sqrt{5} j_{2}(k \beta)$

$-\frac{1}{2 \sqrt{5}}\left[2 j_{1}(k \beta)-3 j_{3}(k \beta)\right]$

$\pm \sqrt{\frac{3}{10}}\left[j_{1}(k \beta)+j_{3}(k \beta)\right]$

$\left[70,2^{-}\right]_{(0,0) ; 1}$

0

$\left[70,2^{+}\right]_{(0,0) ; 2} \quad-\frac{1}{2} \sqrt{15} j_{2}(k \beta) \quad \frac{1}{2} \sqrt{\frac{3}{5}}\left[2 j_{1}(k \beta)-3 j_{3}(k \beta)\right] \quad \mp 3 \sqrt{\frac{1}{10}}\left[j_{1}(k \beta)+j_{3}(k \beta)\right]$

$\left[56,0^{+}\right]_{(1,0) ; 0}-\frac{1-R^{2}}{2 R \sqrt{N}} k \beta j_{1}(k \beta) \quad \frac{1-R^{2}}{6 R \sqrt{N}} k \beta\left[2 j_{0}(k \beta)-j_{2}(k \beta)\right] \quad 0$

$\left[70,0^{+}\right]_{(0,1) ; 0} \quad \frac{\sqrt{1+R^{2}}}{2 R \sqrt{N}} k \beta j_{1}(k \beta) \quad-\frac{\sqrt{1+R^{2}}}{6 R \sqrt{N}} k \beta\left[2 j_{0}(k \beta)-j_{2}(k \beta)\right] \quad 0$ 
Table II: Collective form factors in the large $N$ limit. $H(x)=\arctan x-x /\left(1+x^{2}\right)$. Notation as in Table 1 .

\begin{tabular}{|c|c|c|c|}
\hline Final state & $\mathcal{F}(k)$ & $\mathcal{G}_{0}(k) / m_{3} k_{0} a$ & $\mathcal{G}_{ \pm}(k) / m_{3} k_{0} a$ \\
\hline$\left[56,0^{+}\right]_{(0,0) ; 0}$ & $\frac{1}{\left(1+k^{2} a^{2}\right)^{2}}$ & $\frac{4 k a}{\left(1+k^{2} a^{2}\right)^{3}}$ & 0 \\
\hline$\left[20,1^{+}\right]_{(0,0) ; 0}$ & 0 & 0 & 0 \\
\hline$\left[70,1^{-}\right]_{(0,0) ; 1}$ & $-i \sqrt{3} \frac{k a}{\left(1+k^{2} a^{2}\right)^{2}}$ & $i \sqrt{3} \frac{1-3 k^{2} a^{2}}{\left(1+k^{2} a^{2}\right)^{3}}$ & $\mp i \sqrt{6} \frac{1}{\left(1+k^{2} a^{2}\right)^{2}}$ \\
\hline \multirow[t]{2}{*}[56,2^{+}]{$_{(0,0) ; 0}$} & $\frac{1}{2} \sqrt{5}\left[\frac{-1}{\left(1+k^{2} a^{2}\right)^{2}}\right.$ & $-\frac{1}{2} \sqrt{5}\left[\frac{3+7 k^{2} a^{2}}{k a\left(1+k^{2} a^{2}\right)^{3}}\right.$ & $\pm \sqrt{\frac{15}{2}}\left[\frac{-1}{k a\left(1+k^{2} a^{2}\right)^{2}}\right.$ \\
\hline & $\left.+\frac{3}{2 k^{3} a^{3}} H(k a)\right]$ & $\left.-\frac{9}{2 k^{4} a^{4}} H(k a)\right]$ & $+\frac{3}{2 k^{4} a^{4}} H(k a)$ \\
\hline$\left[70,2^{-}\right]_{(0,0) ; 1}$ & 0 & 0 & 0 \\
\hline \multirow[t]{2}{*}[70,2^{+}]{$_{(0,0) ; 2}$} & $-\frac{1}{2} \sqrt{15}\left[\frac{-1}{\left(1+k^{2} a^{2}\right)^{2}}\right.$ & $\frac{1}{2} \sqrt{15}\left[\frac{3+7 k^{2} a^{2}}{k a\left(1+k^{2} a^{2}\right)^{3}}\right.$ & $\mp \frac{3}{2} \sqrt{10}\left[\frac{-1}{k a\left(1+k^{2} a^{2}\right)^{2}}\right.$ \\
\hline & $\left.+\frac{3}{2 k^{3} a^{3}} H(k a)\right]$ & $\left.-\frac{9}{2 k^{4} a^{4}} H(k a)\right]$ & $+\frac{3}{2 k^{4} a^{4}} H(k a)$ \\
\hline$\left[56,0^{+}\right]_{(1,0) ; 0}$ & $-\frac{1-R^{2}}{R \sqrt{N}} \frac{2 k^{2} a^{2}}{\left(1+k^{2} a^{2}\right)^{3}}$ & $\frac{1-R^{2}}{R \sqrt{N}} \frac{4 k a\left(1-2 k^{2} a^{2}\right)}{\left(1+k^{2} a^{2}\right)^{4}}$ & 0 \\
\hline$\left[70,0^{+}\right]_{(0,1) ; 0}$ & $\frac{\sqrt{1+R^{2}}}{R \sqrt{N}} \frac{2 k^{2} a^{2}}{\left(1+k^{2} a^{2}\right)^{3}}$ & $-\frac{\sqrt{1+R^{2}}}{R \sqrt{N}} \frac{4 k a\left(1-2 k^{2} a^{2}\right)}{\left(1+k^{2} a^{2}\right)^{4}}$ & 0 \\
\hline
\end{tabular}


Table III: Behavior of the collective form factors of Table II for $k a \ll 1$ and $k a \gg 1$.

\begin{tabular}{|c|c|c|c|c|c|c|}
\hline & & $k a \ll 1$ & & & $k a \gg 1$ & \\
\hline Final state & $\mathcal{F}(k)$ & $\mathcal{G}_{0}(k) / m_{3} k_{0} a$ & $\mathcal{G}_{ \pm}(k) / m_{3} k_{0} a$ & $\mathcal{F}(k)$ & $\mathcal{G}_{0}(k) / m_{3} k_{0} a$ & $\mathcal{G}_{ \pm}(k) / m_{3} k_{0} a$ \\
\hline$\left[56,0^{+}\right]_{(0,0) ; 0}$ & $\sim 1$ & $\sim k a$ & 0 & $\sim(k a)^{-4}$ & $\sim(k a)^{-5}$ & 0 \\
\hline$\left[70,1^{-}\right]_{(0,0) ; 1}$ & $\sim k a$ & $\sim 1$ & $\sim 1$ & $\sim(k a)^{-3}$ & $\sim(k a)^{-4}$ & $\sim(k a)^{-4}$ \\
\hline$\left[56,2^{+}\right]_{(0,0) ; 0}$ & $\sim(k a)^{2}$ & $\sim k a$ & $\sim k a$ & $\sim(k a)^{-3}$ & $\sim(k a)^{-4}$ & $\sim(k a)^{-4}$ \\
\hline$\left[70,2^{+}\right]_{(0,0) ; 2}$ & $\sim(k a)^{2}$ & $\sim k a$ & $\sim k a$ & $\sim(k a)^{-3}$ & $\sim(k a)^{-4}$ & $\sim(k a)^{-4}$ \\
\hline$\left[56,0^{+}\right]_{(1,0) ; 0}$ & $\sim(k a)^{2}$ & $\sim k a$ & 0 & $\sim(k a)^{-4}$ & $\sim(k a)^{-5}$ & 0 \\
\hline$\left[70,0^{+}\right]_{(0,1) ; 0}$ & $\sim(k a)^{2}$ & $\sim k a$ & 0 & $\sim(k a)^{-4}$ & $\sim(k a)^{-5}$ & 0 \\
\hline
\end{tabular}


Table IV: Analytic expressions for the transverse proton helicity amplitudes of some nucleon

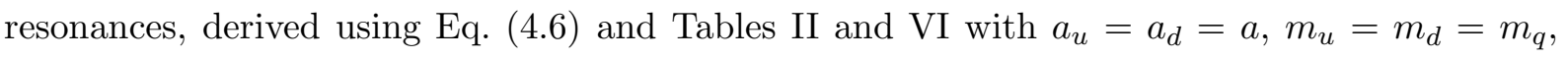
$g_{u}=g_{d}=g$ and $\mu_{u}=\mu_{d}=\mu . \quad Z(x)=-\frac{1}{\left(1+x^{2}\right)^{2}}+\frac{3}{2 x^{3}} H(x)$ with $H(x)=\arctan x-\frac{x}{\left(1+x^{2}\right)}$ and $\chi=\left(1-R^{2}\right) / R \sqrt{N}$. $\zeta$ denotes an additional multiplicative sign-factor in accord with the convention explained in the text.

\begin{tabular}{|c|c|c|c|c|}
\hline Resonance & State & $\nu$ & $A_{\nu}^{p}$ & $\zeta$ \\
\hline$N(1535) S_{11}$ & ${ }^{2} 8_{1 / 2}\left[70,1^{-}\right]_{(0,0) ; 1}$ & $1 / 2$ & $i \sqrt{2} \sqrt{\frac{\pi}{k_{0}}} \mu \frac{1}{\left(1+k^{2} a^{2}\right)^{2}}\left[2 \frac{m_{q} k_{0} a}{g}+k^{2} a\right]$ & +1 \\
\hline \multirow[t]{2}{*}{$N(1520) D_{13}$} & ${ }^{2} 8_{3 / 2}\left[70,1^{-}\right]_{(0,0) ; 1}$ & $1 / 2$ & $2 i \sqrt{\frac{\pi}{k_{0}}} \mu \frac{1}{\left(1+k^{2} a^{2}\right)^{2}}\left[\frac{m_{q} k_{0} a}{g}-k^{2} a\right]$ & +1 \\
\hline & & $3 / 2$ & $2 i \sqrt{3} \sqrt{\frac{\pi}{k_{0}}} \mu \frac{1}{\left(1+k^{2} a^{2}\right)^{2}} \frac{m_{q} k_{0} a}{g}$ & +1 \\
\hline$N(1650) S_{11}$ & ${ }^{4} 8_{1 / 2}\left[70,1^{-}\right]_{(0,0) ; 1}$ & $1 / 2$ & 0 & \\
\hline$N(1700) D_{13}$ & ${ }^{4} 8_{3 / 2}\left[70,1^{-}\right]_{(0,0) ; 1}$ & $1 / 2,3 / 2$ & 0 & \\
\hline$N(1675) D_{15}$ & ${ }^{4} 8_{5 / 2}\left[70,1^{-}\right]_{(0,0) ; 1}$ & $1 / 2,3 / 2$ & 0 & \\
\hline \multirow[t]{2}{*}{$N(1720) P_{13}$} & ${ }^{2} 8_{3 / 2}\left[56,2^{+}\right]_{(0,0) ; 0}$ & $1 / 2$ & $-\sqrt{2} \sqrt{\frac{\pi}{k_{0}}} \mu\left[3 \frac{m_{q} k_{0} a}{g}+k^{2} a\right] \frac{1}{k a} Z(k a)$ & -1 \\
\hline & & $3 / 2$ & $\sqrt{6} \sqrt{\frac{\pi}{k_{0}}} \mu \frac{m_{q} k_{0} a}{g} \frac{1}{k a} Z(k a)$ & -1 \\
\hline \multirow[t]{2}{*}{$N(1680) F_{15}$} & ${ }^{2} 8_{5 / 2}\left[56,2^{+}\right]_{(0,0) ; 0}$ & $1 / 2$ & $-\sqrt{3} \sqrt{\frac{\pi}{k_{0}}} \mu\left[2 \frac{m_{q} k_{0} a}{g}-k^{2} a\right] \frac{1}{k a} Z(k a)$ & -1 \\
\hline & & $3 / 2$ & $-2 \sqrt{6} \sqrt{\frac{\pi}{k_{0}}} \mu \frac{m_{q} k_{0} a}{g} \frac{1}{k a} Z(k a)$ & -1 \\
\hline$N(1440) P_{11}$ & ${ }^{2} 8_{1 / 2}\left[56,0^{+}\right]_{(1,0) ; 0}$ & $1 / 2$ & $-2 \chi \sqrt{\frac{\pi}{k_{0}}} \mu k \frac{2 k^{2} a^{2}}{\left(1+k^{2} a^{2}\right)^{3}}$ & -1 \\
\hline
\end{tabular}


Table V: Analytic expressions for the transverse helicity amplitudes of some delta resonances resonances, derived using Eq. (4.6) and Tables \and VII with $a_{u}=a_{d}=a, m_{u}=m_{d}=m_{q}$, $g_{u}=g_{d}=g$ and $\mu_{u}=\mu_{d}=\mu$. Notation as in Table IV.

\begin{tabular}{|c|c|c|c|c|}
\hline Resonance & State & $\nu$ & $A_{\nu}^{p}=A_{\nu}^{n}$ & $\zeta$ \\
\hline \multirow[t]{2}{*}{$\Delta(1232) P_{33}$} & ${ }^{4} 10_{3 / 2}\left[56,0^{+}\right]_{(0,0) ; 0}$ & $1 / 2$ & $-\frac{2 \sqrt{2}}{3} \sqrt{\frac{\pi}{k_{0}}} \mu k \frac{1}{\left(1+k^{2} a^{2}\right)^{2}}$ & +1 \\
\hline & & $3 / 2$ & $-\frac{2 \sqrt{2}}{\sqrt{3}} \sqrt{\frac{\pi}{k_{0}}} \mu k \frac{1}{\left(1+k^{2} a^{2}\right)^{2}}$ & +1 \\
\hline$\Delta(1620) S_{31}$ & ${ }^{2} 10_{1 / 2}\left[70,1^{-}\right]_{(0,0) ; 1}$ & $1 / 2$ & $-i \frac{\sqrt{2}}{3} \sqrt{\frac{\pi}{k_{0}}} \mu \frac{1}{\left(1+k^{2} a^{2}\right)^{2}}\left[6 \frac{m_{q} k_{0} a}{g}-k^{2} a\right]$ & -1 \\
\hline \multirow[t]{2}{*}{$\Delta(1700) D_{33}$} & ${ }^{2} 10_{3 / 2}\left[70,1^{-}\right]_{(0,0) ; 1}$ & $1 / 2$ & $-i \frac{2}{3} \sqrt{\frac{\pi}{k_{0}}} \mu \frac{1}{\left(1+k^{2} a^{2}\right)^{2}}\left[3 \frac{m_{q} k_{0} a}{g}+k^{2} a\right]$ & -1 \\
\hline & & $3 / 2$ & $-2 i \sqrt{3} \sqrt{\frac{\pi}{k_{0}}} \mu \frac{1}{\left(1+k^{2} a^{2}\right)^{2}} \frac{m_{q} k_{0} a}{g}$ & -1 \\
\hline \multirow[t]{2}{*}{$\Delta(1600) P_{33}$} & ${ }^{4} 10_{3 / 2}\left[56,0^{+}\right]_{(1,0) ; 0}$ & $1 / 2$ & $\frac{2 \sqrt{2}}{3} \chi \sqrt{\frac{\pi}{k_{0}}} \mu k \frac{2 k^{2} a^{2}}{\left(1+k^{2} a^{2}\right)^{3}}$ & +1 \\
\hline & & $3 / 2$ & $\frac{2 \sqrt{2}}{\sqrt{3}} \chi \sqrt{\frac{\pi}{k_{0}}} \mu k \frac{2 k^{2} a^{2}}{\left(1+k^{2} a^{2}\right)^{3}}$ & +1 \\
\hline
\end{tabular}


Table VI: Orbit- and spin-flip amplitudes of Eq. (4.7), associated with transverse helicity amplitudes for nucleon resonances (proton-target couplings) according to Eq. (4.6). $y_{i}=x_{i} / g_{i}$ and $x_{i}=\mu_{i} e_{i} ; \mathcal{F}_{i}(k), \mathcal{G}_{+, i}$ are obtained from the corresponding entries in Table II with $a \rightarrow a_{i}$, $m_{3} \rightarrow m_{i}$ with $i=u, d$. Neutron-target couplings are obtained by interchanging $u \leftrightarrow d$.

\begin{tabular}{|c|c|c|c|}
\hline \multirow[b]{2}{*}{ State } & \multirow[b]{2}{*}{$\nu$} & \multicolumn{2}{|c|}{$A_{\nu}^{p}$} \\
\hline & & $\mathcal{A}$ & $\mathcal{B}$ \\
\hline \multirow[t]{2}{*}{$28[56]$} & $1 / 2$ & $\frac{1}{3}\left[2 y_{u} \mathcal{G}_{u,+}(k)+y_{d} \mathcal{G}_{d,+}(k)\right]$ & $\frac{1}{9}\left[4 x_{u} \mathcal{F}_{u}(k)-x_{d} \mathcal{F}_{d}(k)\right]$ \\
\hline & $3 / 2$ & $\frac{1}{3}\left[2 y_{u} \mathcal{G}_{u,+}(k)+y_{d} \mathcal{G}_{d,+}(k)\right]$ & 0 \\
\hline \multirow[t]{2}{*}{${ }^{2} 8[70]$} & $1 / 2$ & $\frac{1}{3 \sqrt{2}}\left[y_{u} \mathcal{G}_{u,+}(k)-y_{d} \mathcal{G}_{d,+}(k)\right]$ & $\frac{1}{9 \sqrt{2}}\left[5 x_{u} \mathcal{F}_{u}(k)+x_{d} \mathcal{F}_{d}(k)\right]$ \\
\hline & $3 / 2$ & $\frac{1}{3 \sqrt{2}}\left[y_{u} \mathcal{G}_{u,+}(k)-y_{d} \mathcal{G}_{d,+}(k)\right]$ & 0 \\
\hline \multirow[t]{2}{*}{$28[20]$} & $1 / 2$ & 0 & 0 \\
\hline & $3 / 2$ & 0 & 0 \\
\hline \multirow[t]{2}{*}{${ }^{4} 8[70]$} & $1 / 2$ & 0 & $\frac{1}{9 \sqrt{2}}\left[x_{u} \mathcal{F}_{u}(k)+2 x_{d} \mathcal{F}_{d}(k)\right]$ \\
\hline & $3 / 2$ & 0 & $\frac{1}{3 \sqrt{6}}\left[x_{u} \mathcal{F}_{d}(k)+2 x_{d} \mathcal{F}_{d}(k)\right]$ \\
\hline
\end{tabular}


Table VII: Orbit- and spin-flip amplitudes of Eq. (4.7), associated with transverse helicity amplitudes for delta resonances. Notation as in Table $\mathrm{VI}$.

\begin{tabular}{|c|c|c|c|}
\hline \multirow[b]{2}{*}{ State } & \multirow[b]{2}{*}{$\nu$} & \multicolumn{2}{|c|}{$A_{\nu}^{p}=A_{\nu}^{n}$} \\
\hline & & $\mathcal{A}$ & $\mathcal{B}$ \\
\hline \multirow[t]{2}{*}{${ }^{2} 10[70]$} & $1 / 2$ & $\frac{-1}{3 \sqrt{2}}\left[y_{u} \mathcal{G}_{u,+}(k)-y_{d} \mathcal{G}_{d,+}(k)\right]$ & $\frac{1}{9 \sqrt{2}}\left[x_{u} \mathcal{F}_{u}(k)-x_{d} \mathcal{F}_{d}(k)\right]$ \\
\hline & $3 / 2$ & $\frac{-1}{3 \sqrt{2}}\left[y_{u} \mathcal{G}_{u,+}(k)-y_{d} \mathcal{G}_{d,+}(k)\right]$ & 0 \\
\hline \multirow[t]{2}{*}{${ }^{4} 10[56]$} & $1 / 2$ & 0 & $\frac{-\sqrt{2}}{9}\left[x_{u} \mathcal{F}_{u}(k)-x_{d} \mathcal{F}_{d}(k)\right]$ \\
\hline & $3 / 2$ & 0 & $\frac{-\sqrt{2}}{3 \sqrt{3}}\left[x_{u} \mathcal{F}_{u}(k)-x_{d} \mathcal{F}_{d}(k)\right]$ \\
\hline
\end{tabular}




\section{Figure Captions}

Figure 1: Collective model of baryons and its idealized string configuration (the charge distribution of the proton is shown as an example).

Figure 2: Comparison between the experimental neutron electric form factor $G_{E}^{n}$, and the collective form factor with and without flavor breaking (dashed and solid lines). The experimental data, taken from [28], and the calculations are divided by the dipole form factor, $F_{D}=1 /\left(1+Q^{2} / 0.71\right)^{2}$.

Figure 3: Comparison between the experimental proton electric form factor $G_{E}^{p}$, and the collective form factor with and without flavor breaking (dashed and solid lines). The experimental data taken from [27], and the calculations are divided by the dipole form factor, $F_{D}=1 /\left(1+Q^{2} / 0.71\right)^{2}$.

Figure 4: Comparison between the experimental neutron magnetic form factor $G_{M}^{n}$, and the collective form factor with and without flavor breaking (dashed and solid lines). The

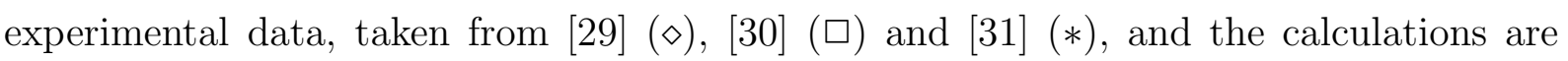
divided by $\mu_{n} F_{D}$.

Figure 5: Comparison between the experimental proton magnetic form factor $G_{M}^{p}$, and the collective form factor with and without flavor breaking (dashed and solid lines). The experimental data, taken from [27], and the calculations are divided by $\mu_{p} F_{D}$.

Figure 6: Comparison between the experimental ratio of the neutron and proton magnetic form factors and the calculated ratio using the collective form factors with and without flavor breaking (dashed and solid lines). The experimental values are from 229, 32 $(\diamond)$, [30, 27] (口) and [31, 27] $(*)$.

Figure 7: Proton helicity amplitudes for excitation of $N(1520) D_{13}$ (a factor of $+i$ is suppressed). The calculation with and without flavor breaking are shown by dashed and 
solid lines, respectively. The experimental data are from [34.

Figure 8: Same as Figure 7, but for $N(1535) S_{11}$ (a factor of $+i$ is suppressed).

Figure 9: Same as Figure 7, but for $N(1650) S_{11}$ (a factor of $+i$ is suppressed).

Figure 10: Same as Figure 7, but for $N(1680) F_{15}$.

Figure 11: Neutron helicity amplitudes for $N(1680) F_{15}$.

Figure 12: Helicity amplitudes for the excitation of $\Delta(1232) P_{33}$. The calculation with and without flavor breaking are shown by dashed and solid lines, respectively. Only the data at $Q^{2}=0$ (photoproduction) 33 are shown, since the other experimental results have not been analyzed in terms of helicity amplitudes.

Figure 13: Helicity-1/2 amplitude for excitation of $\Delta(1620) S_{31}$ (a factor of $+i$ is suppressed). The calculation with and without flavor breaking are shown by dashed and solid lines, respectively. The experimental data are from [33] and [34].

Figure 14: Same as Figure 13, but for $\Delta(1700) D_{33}$ (a factor of $+i$ is suppressed).

Figure 15: Same as Figure 13, but for helicity-3/2 amplitude of $\Delta(1700) D_{33}$ (a factor of $+i$ is suppressed).

Figure 16: Helicity asymmetry for $\Delta(1232) P_{33}$. The experimental data are from [33].

Figure 17: Proton helicity asymmetry for $N(1520) D_{13}$. The experimental data are from [34.

Figure 18: Same as Figure 17, but for $N(1680) F_{15}$.

Figure 19: Proton helicity amplitude for excitation of $N(1440) P_{11}$. The calculation with and without flavor breaking are shown by dashed and solid lines, respectively. The curves 
are labelled by the value of $\chi$ (see Table [V). The experimental data are from [34].

Figure 20: Effect of hadron swelling for excitation of $\Delta(1232) P_{33}$. The curves are labelled by the value of the stretching parameter $\xi$ of Eq. (6.1).

Figure 21: Same as Figure 20, but for $N(1520) D_{13}$ (a factor of $+i$ is suppressed).

Figure 22: Same as Figure 20, but for $N(1680) F_{15}$. 

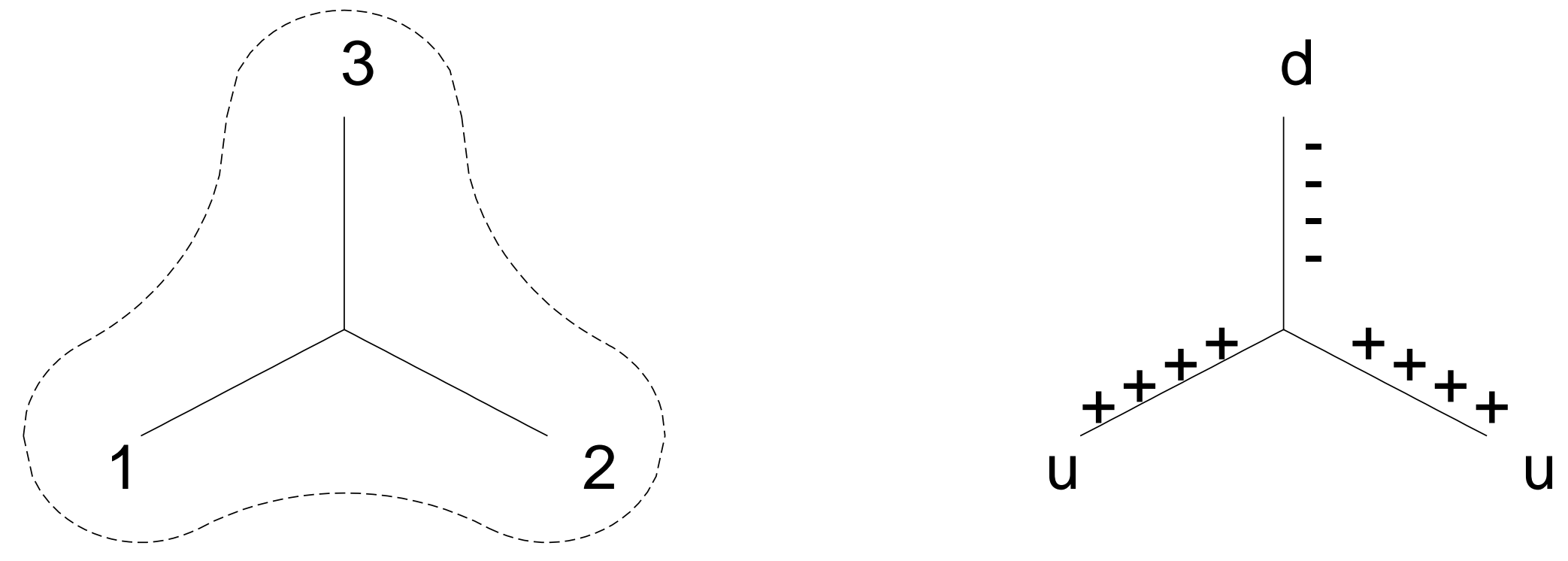


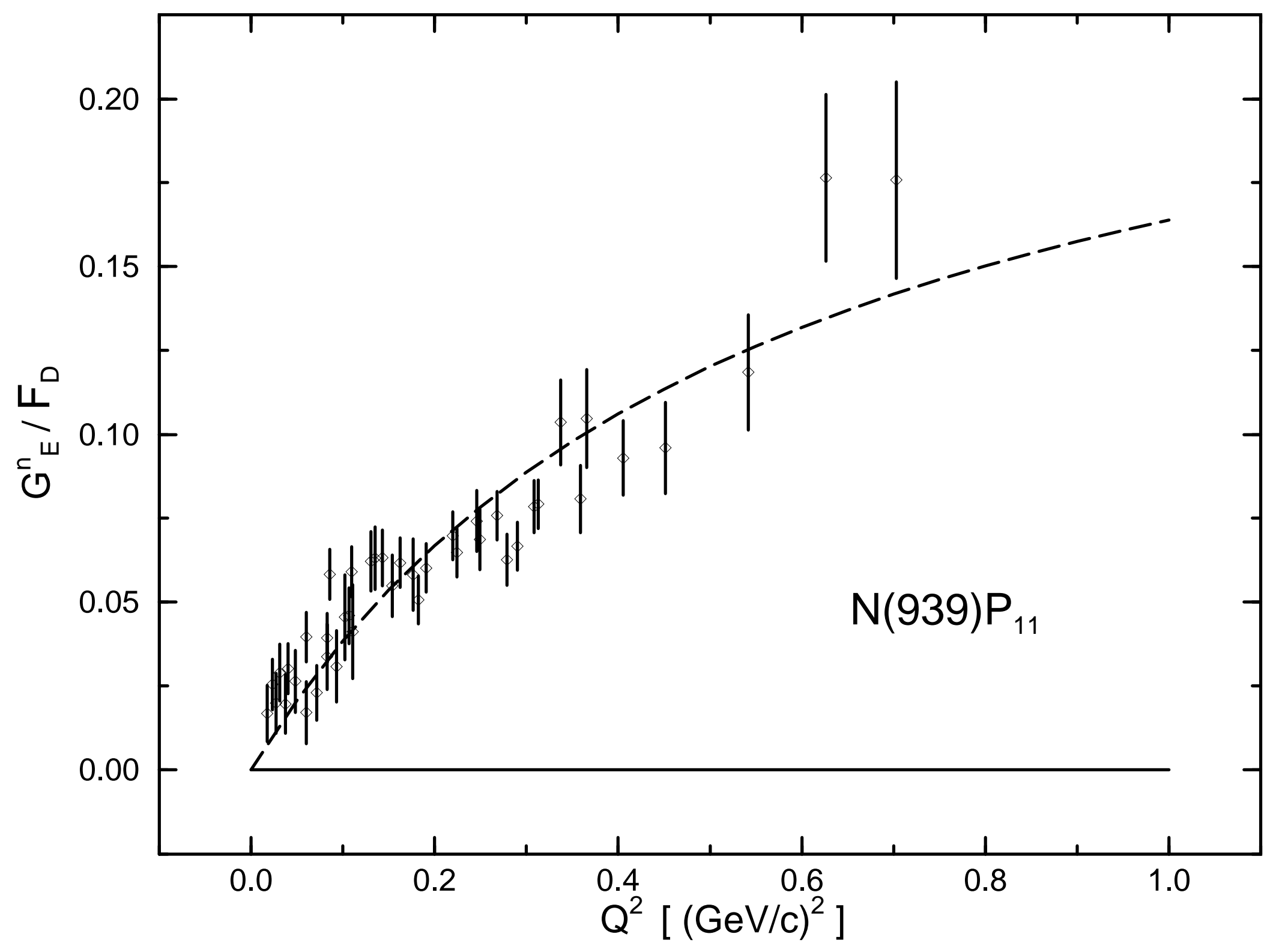




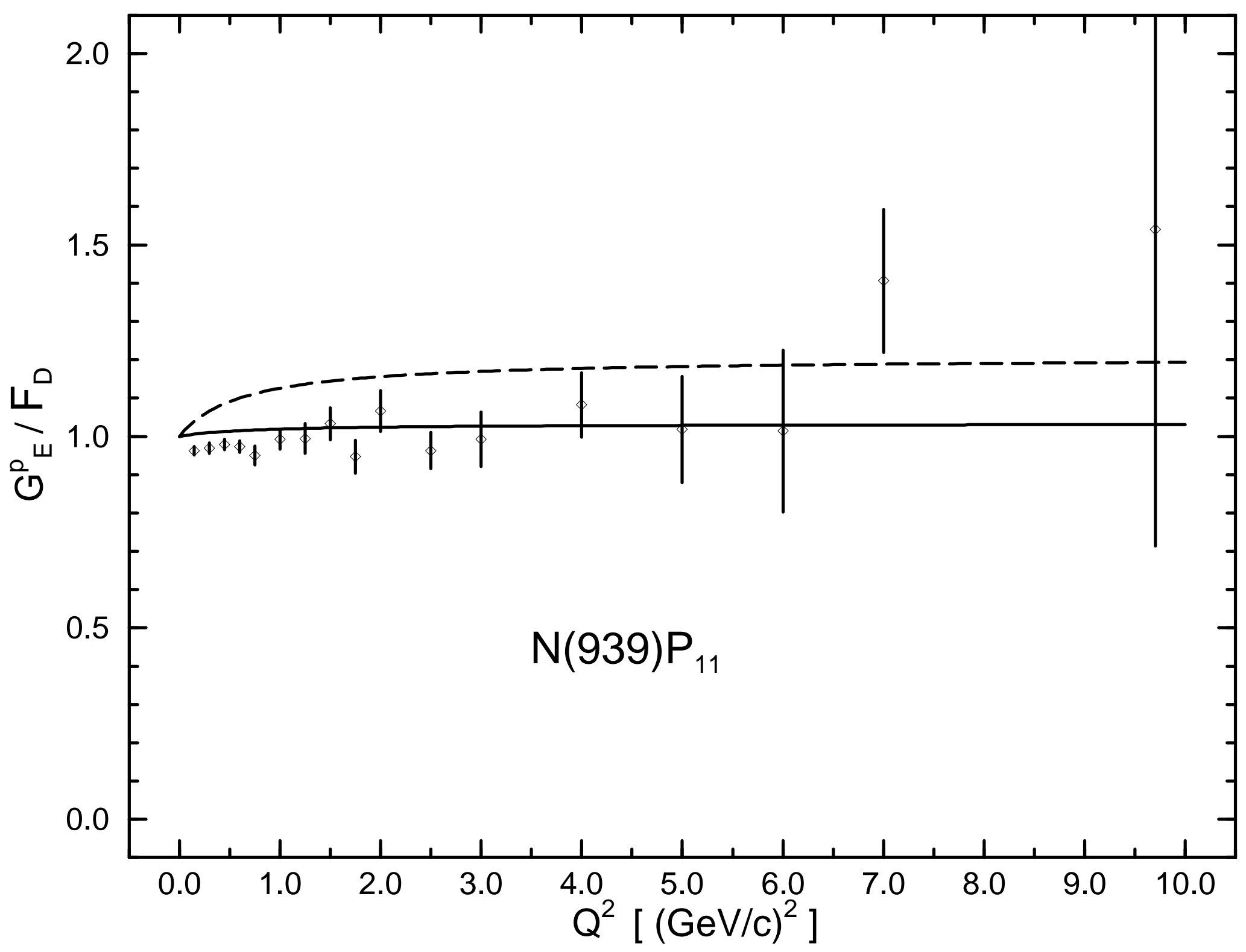




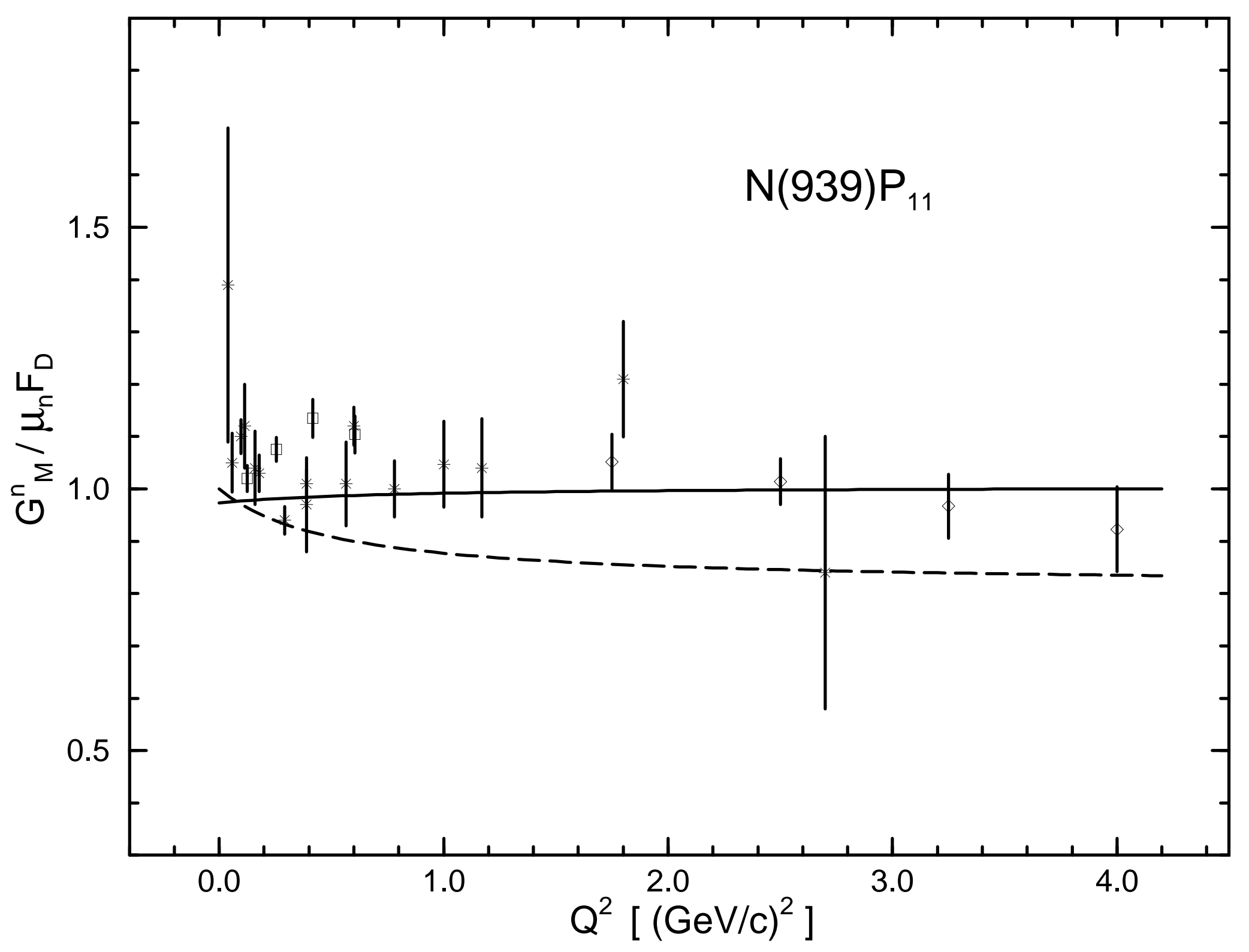




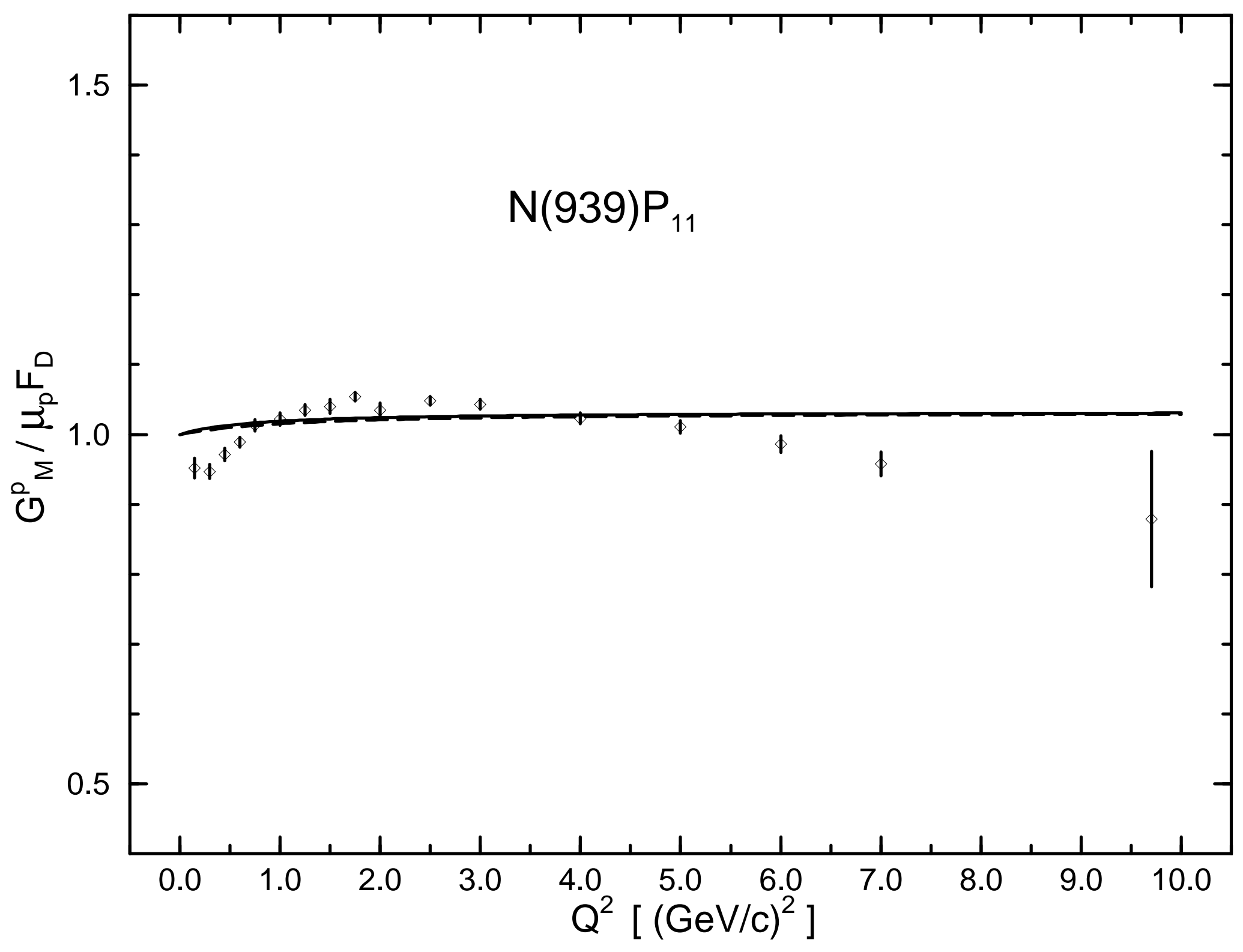




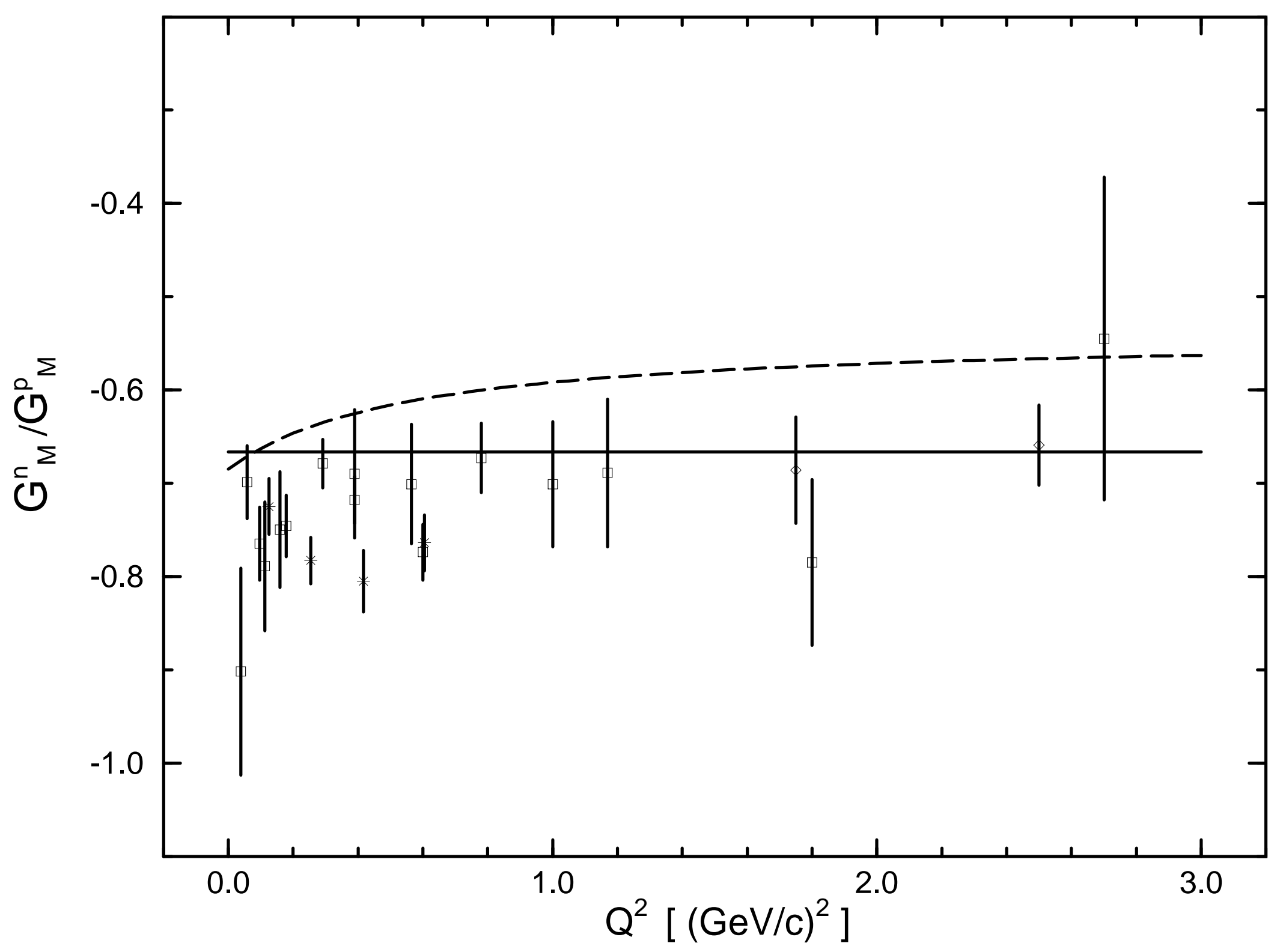




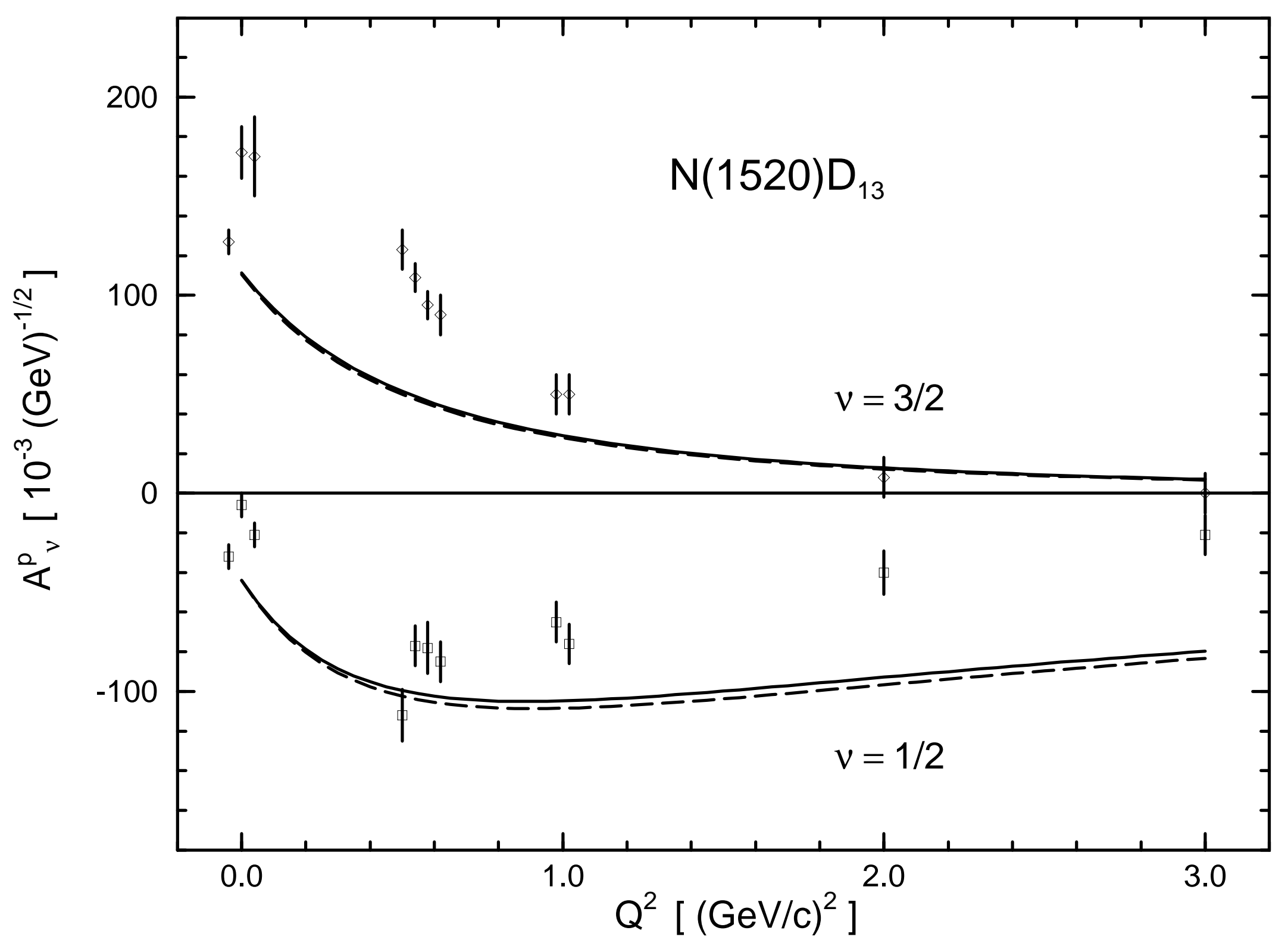




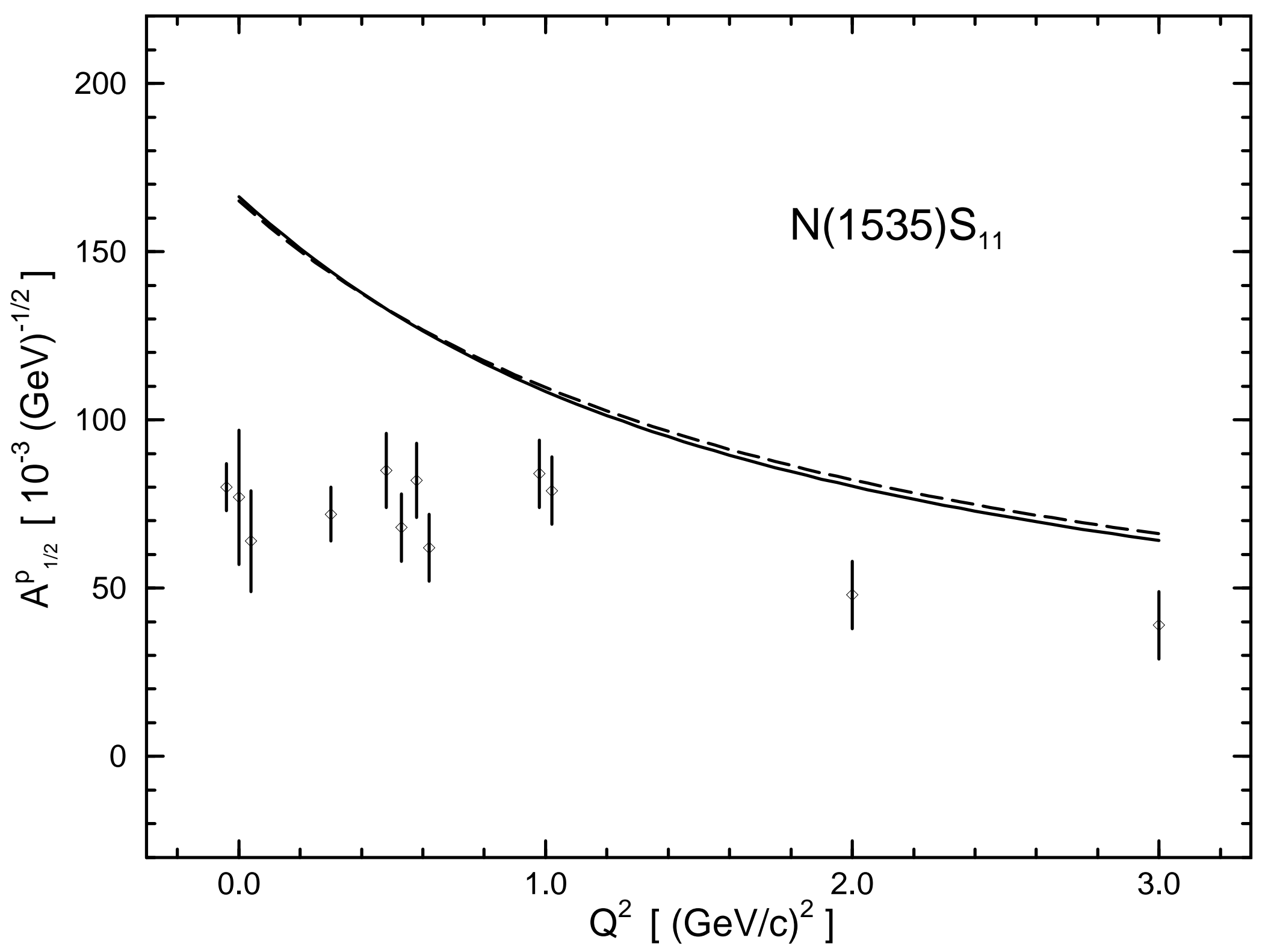




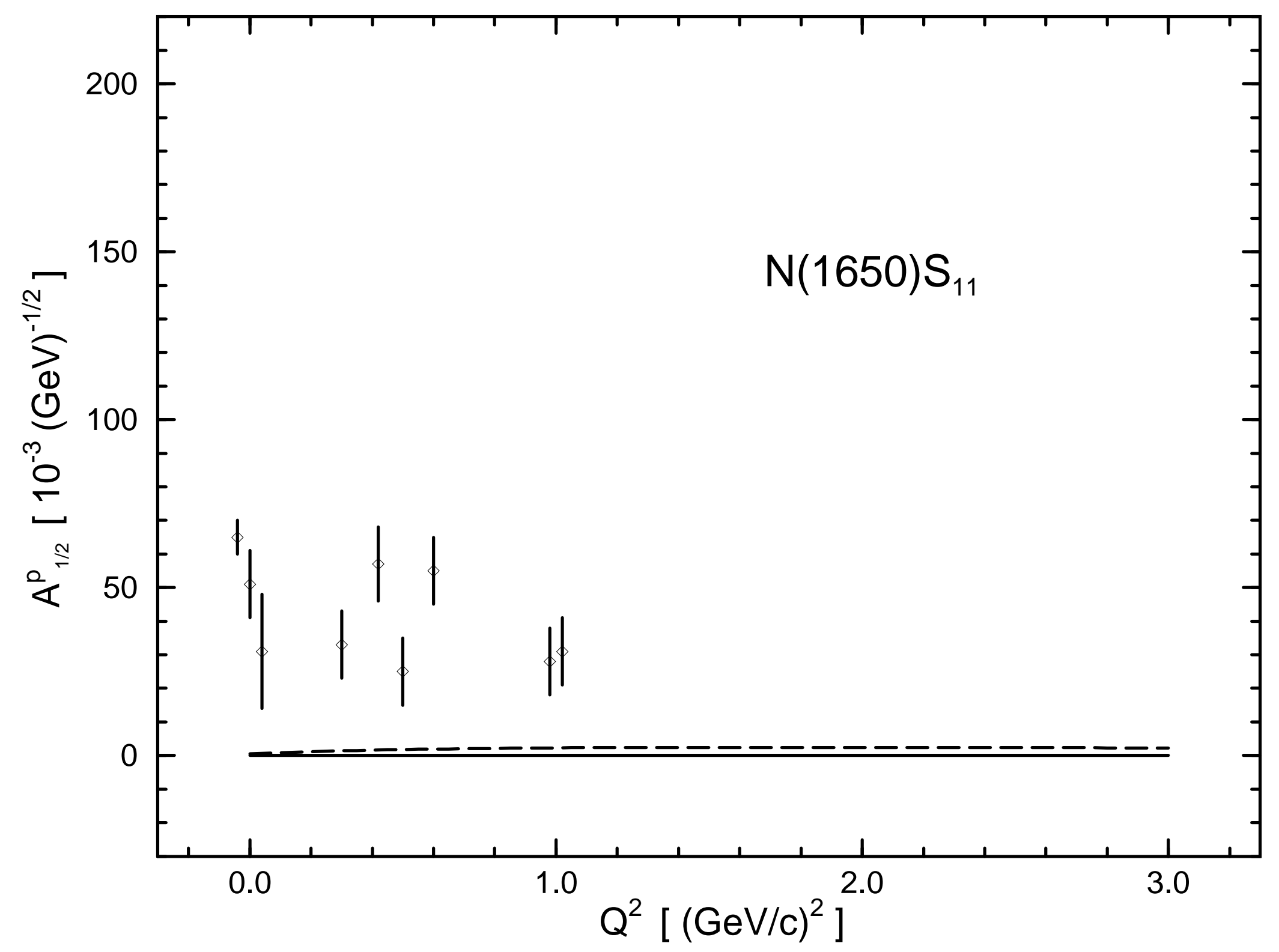




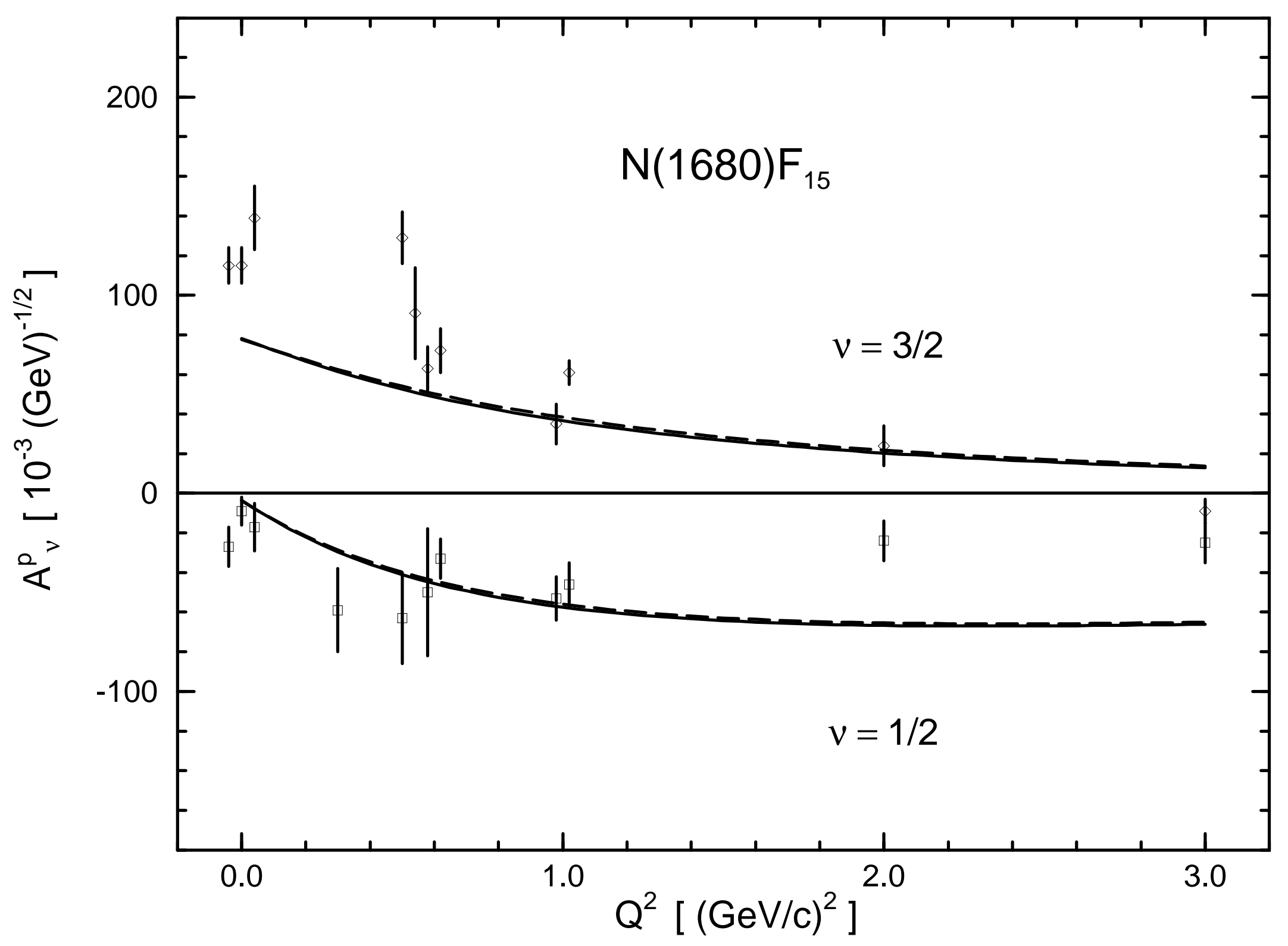




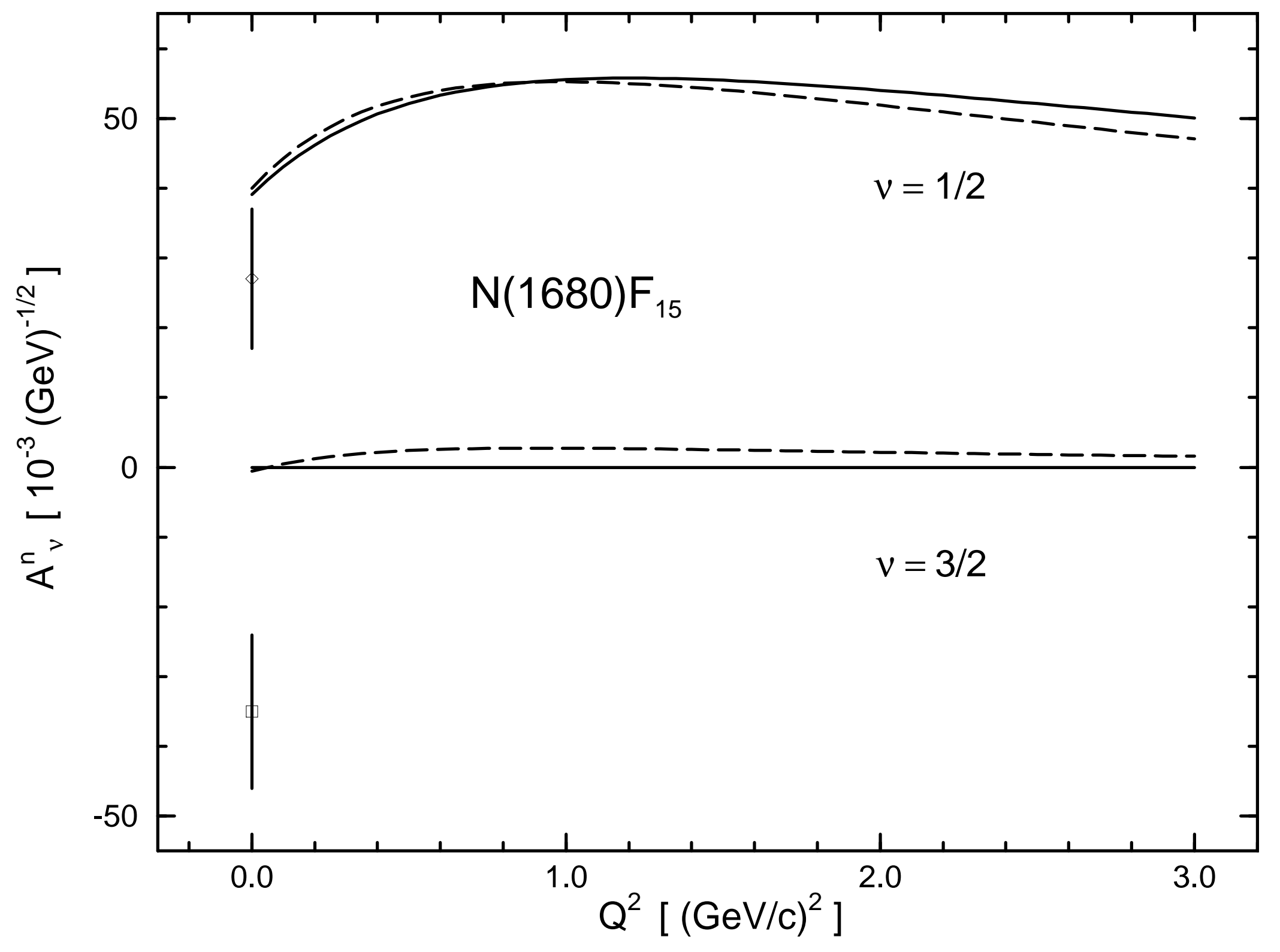




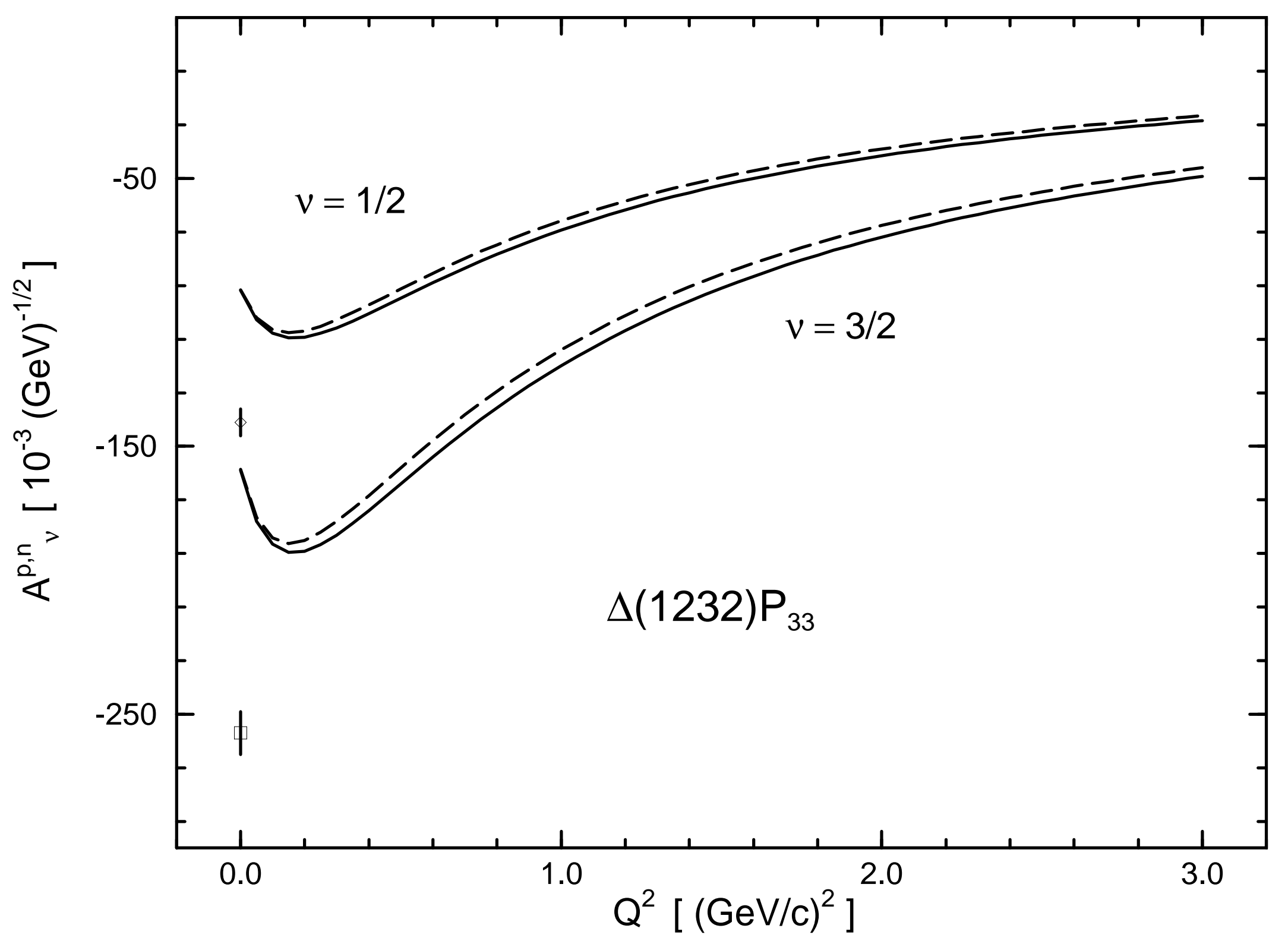




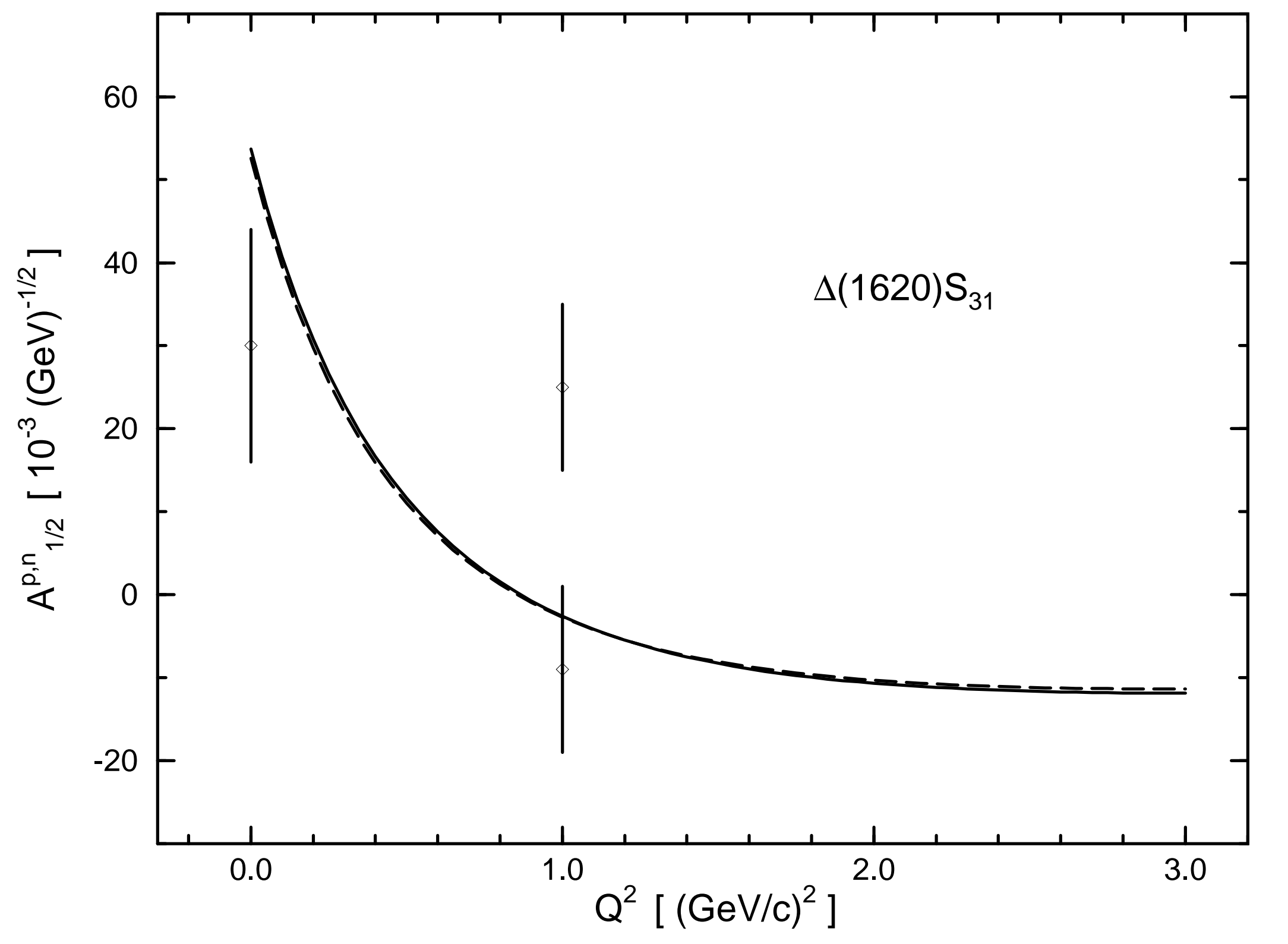




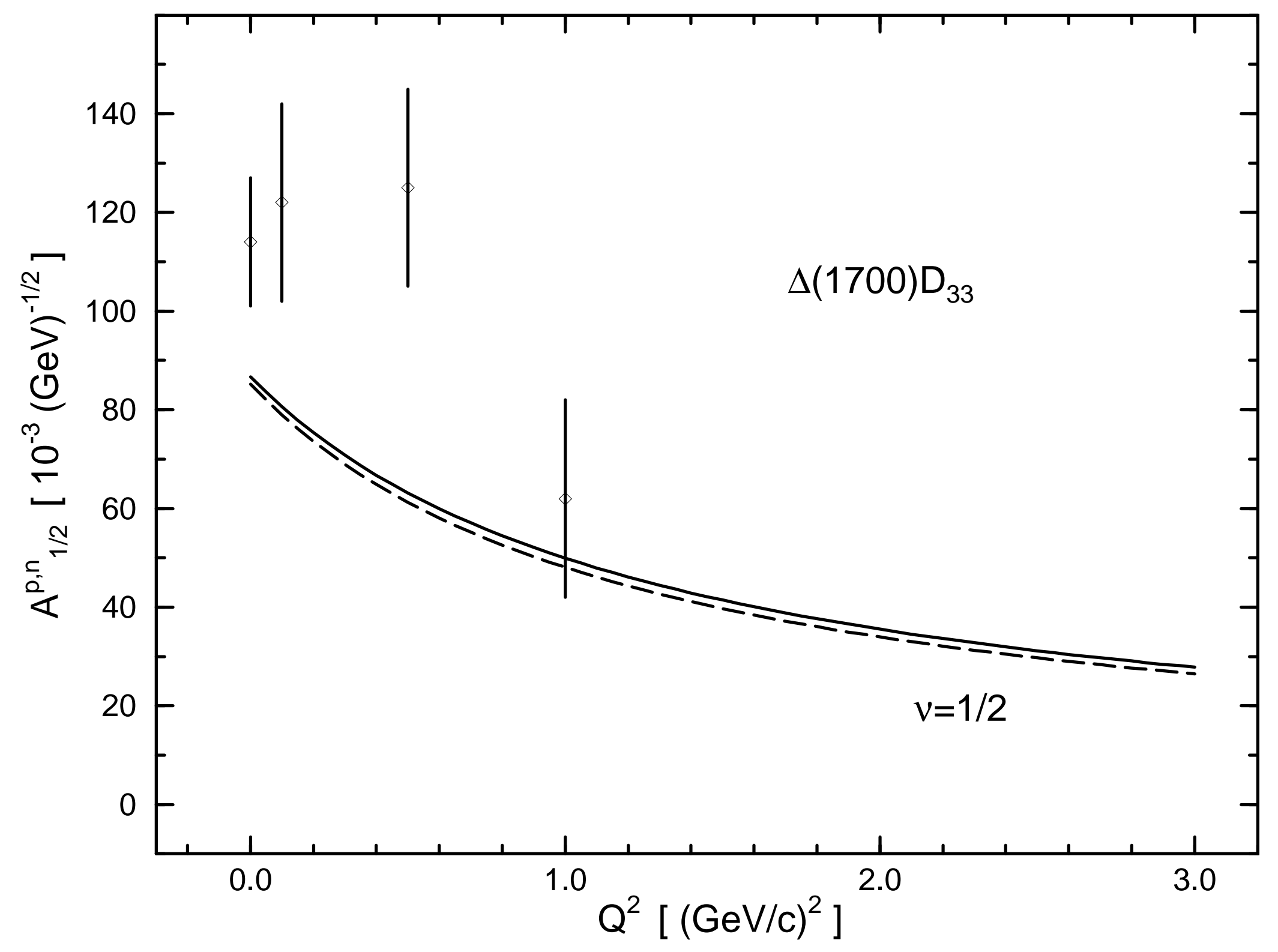




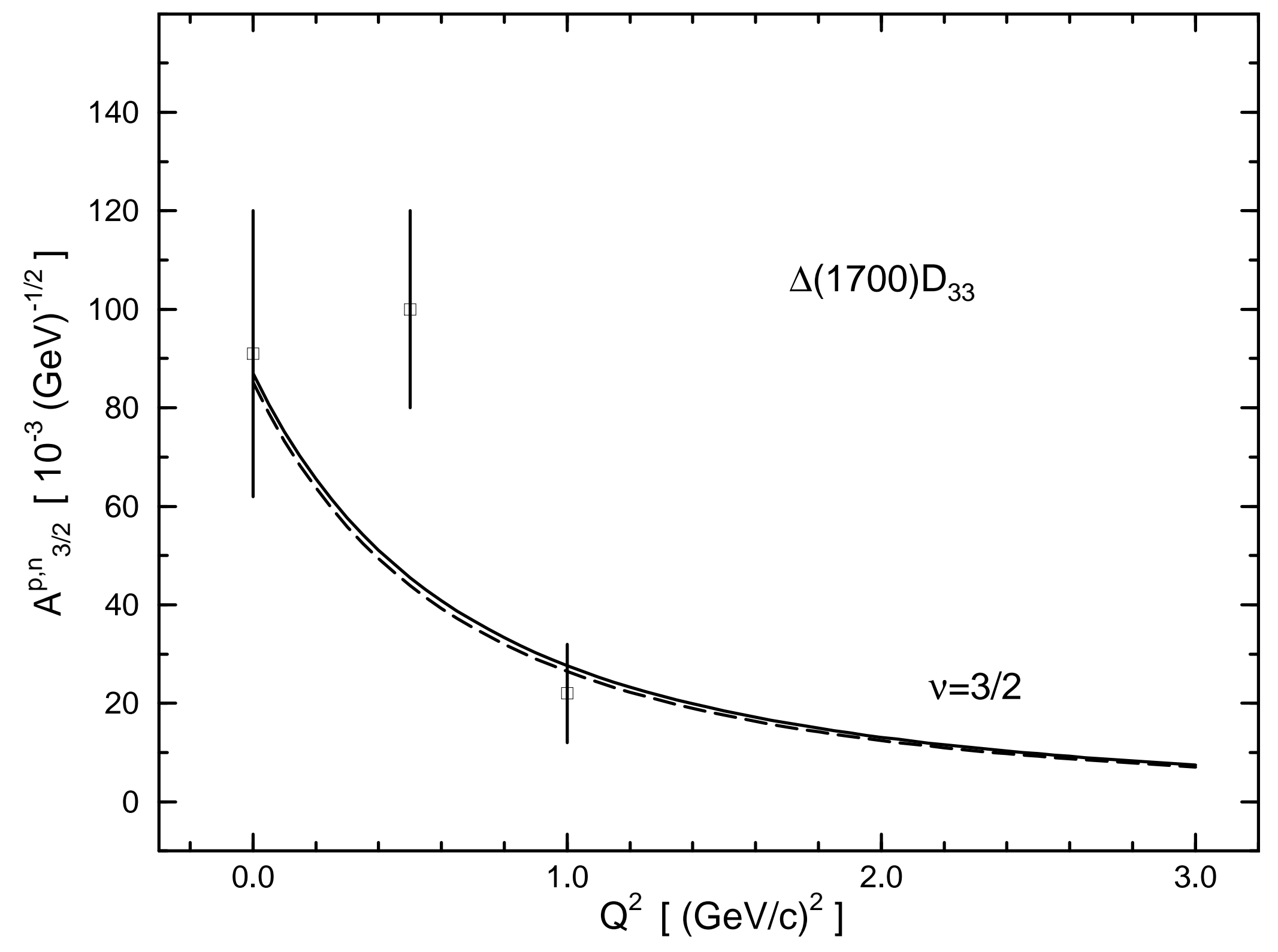




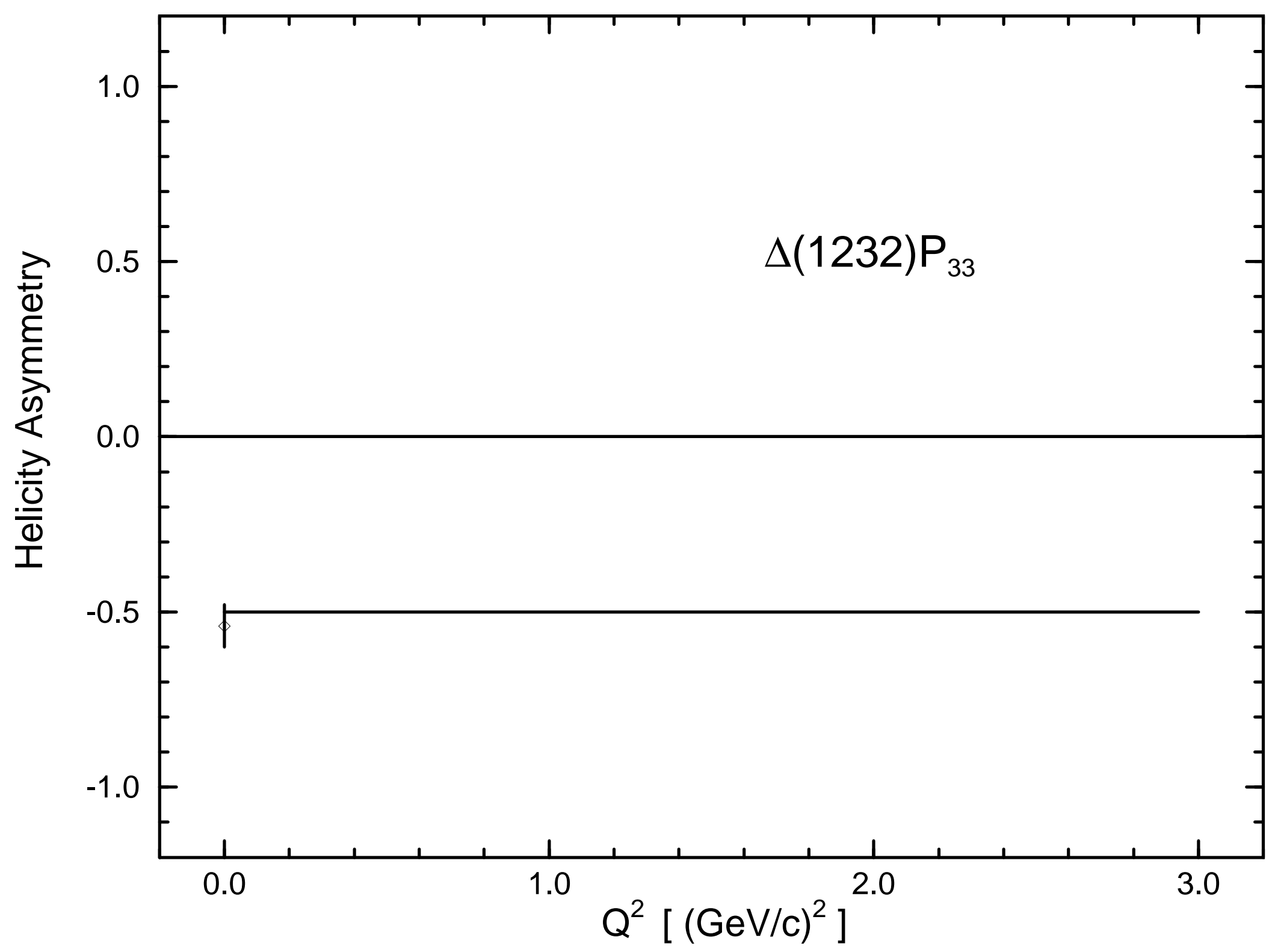




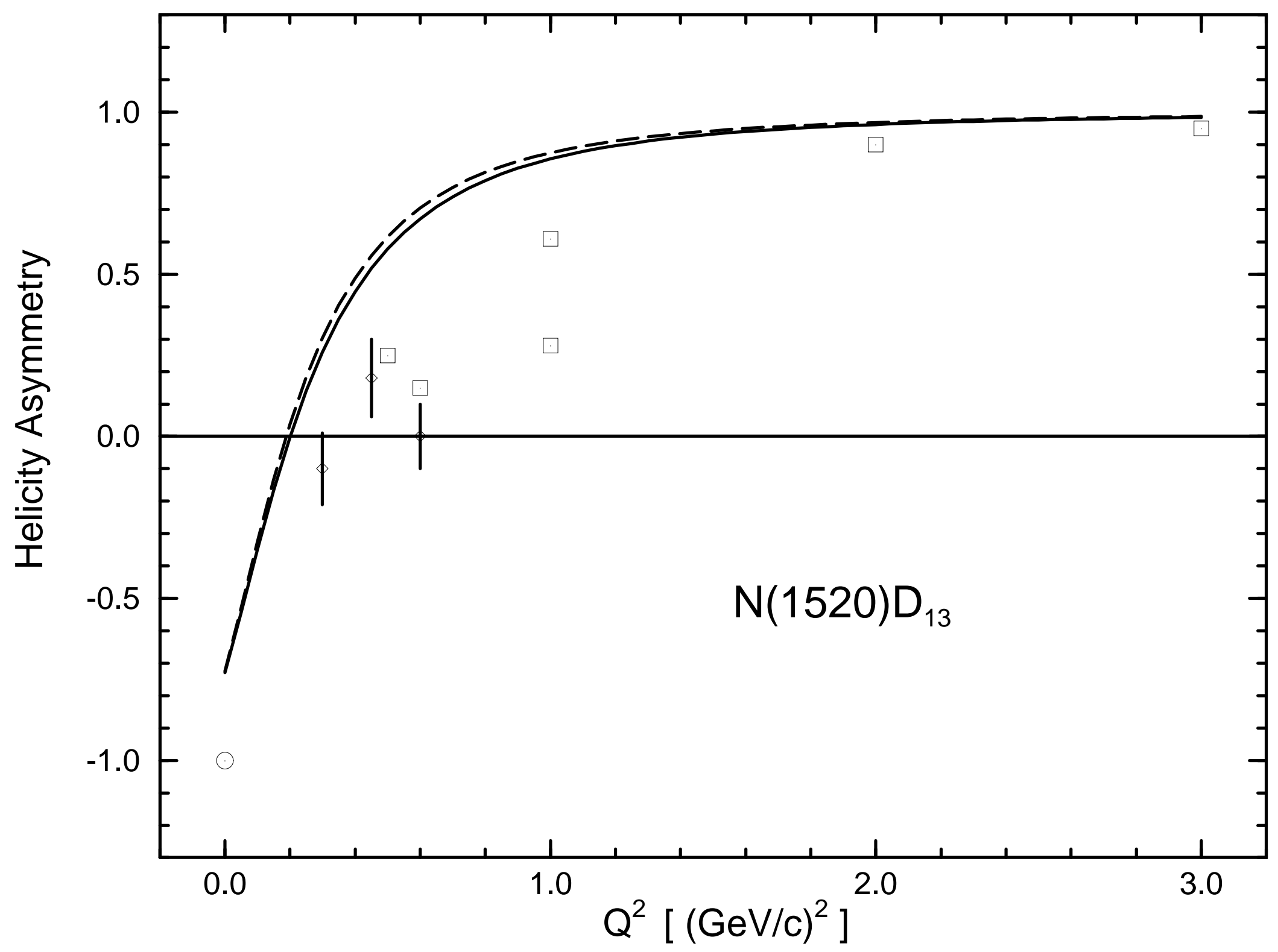




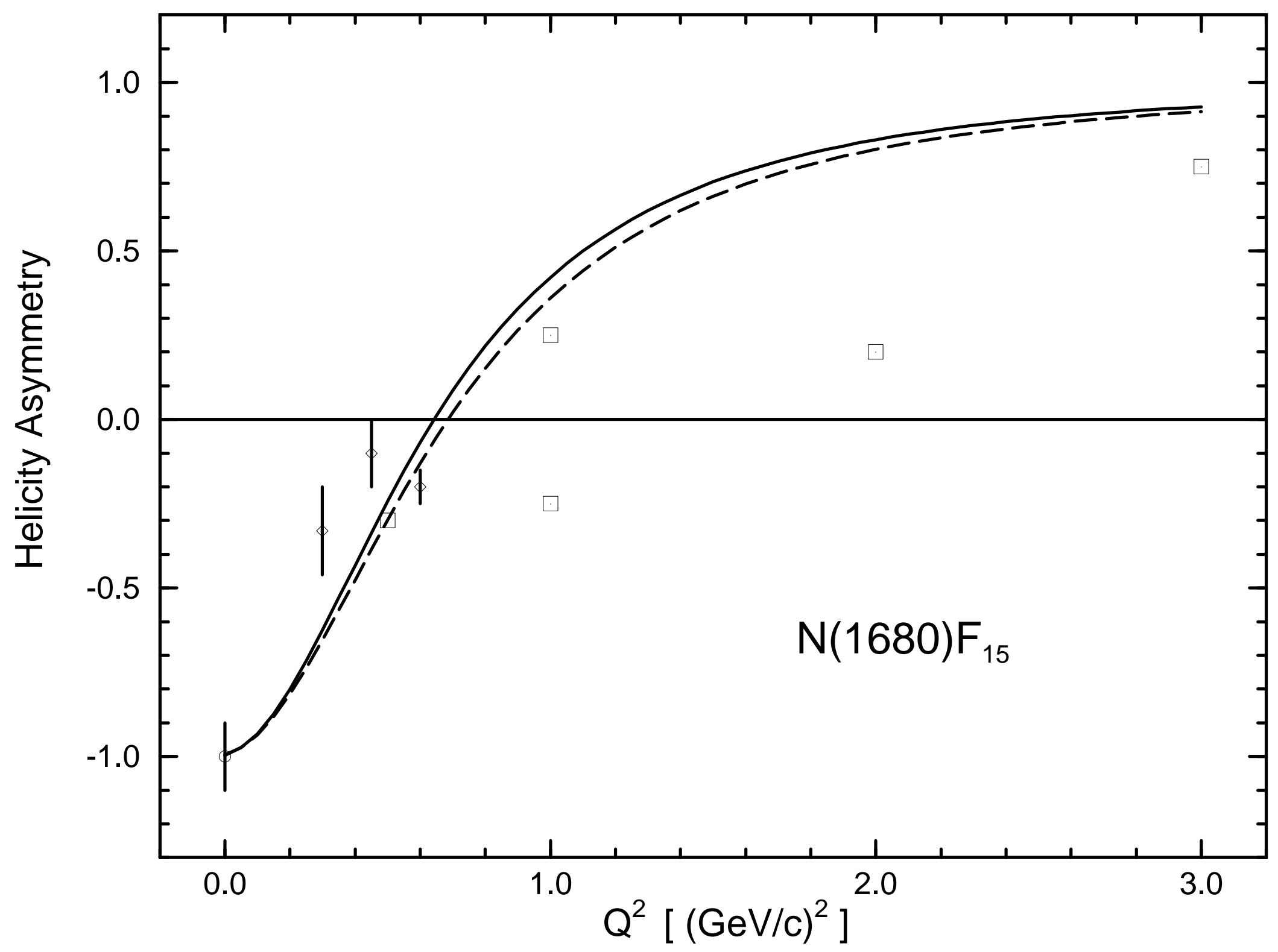




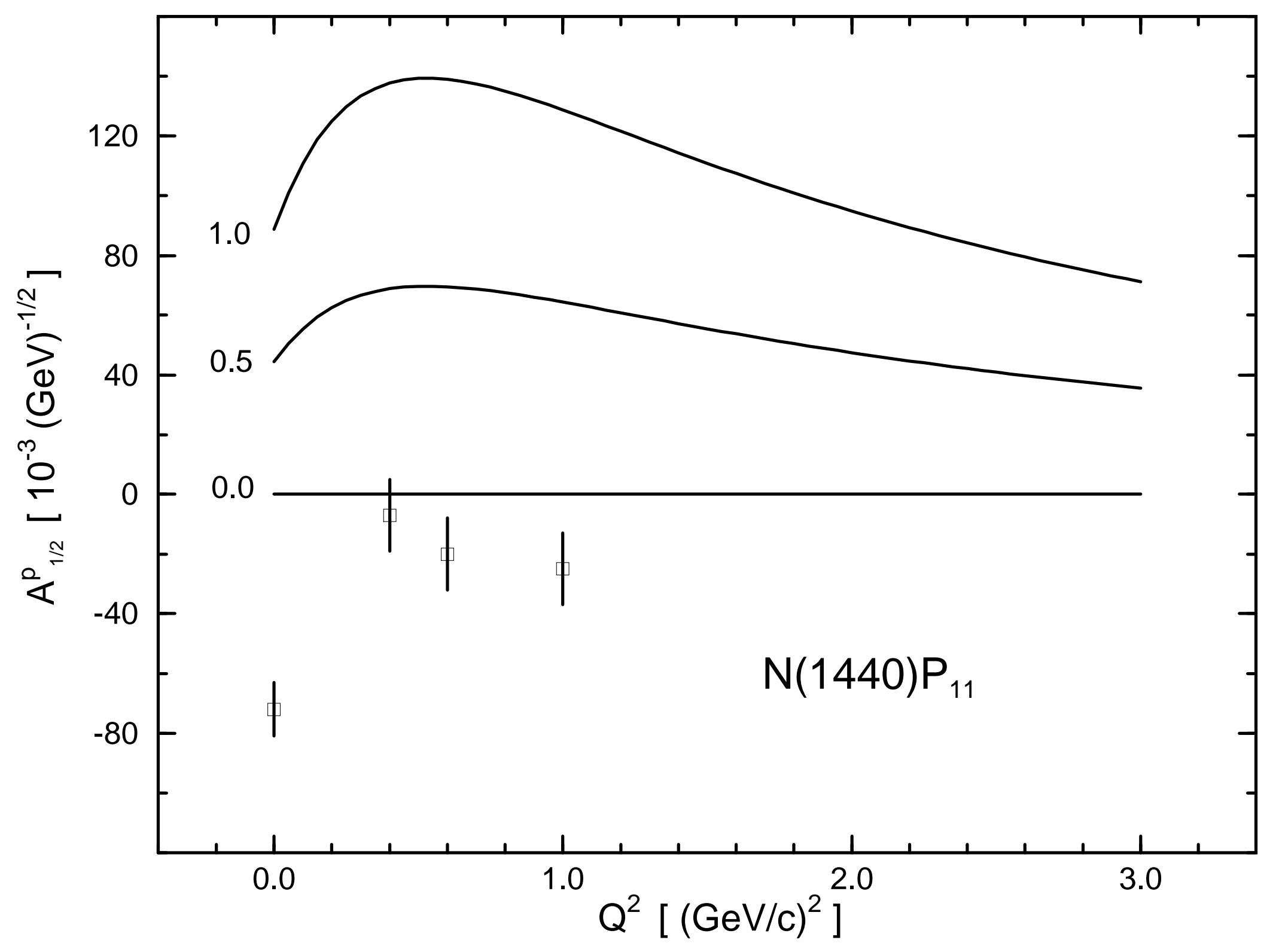




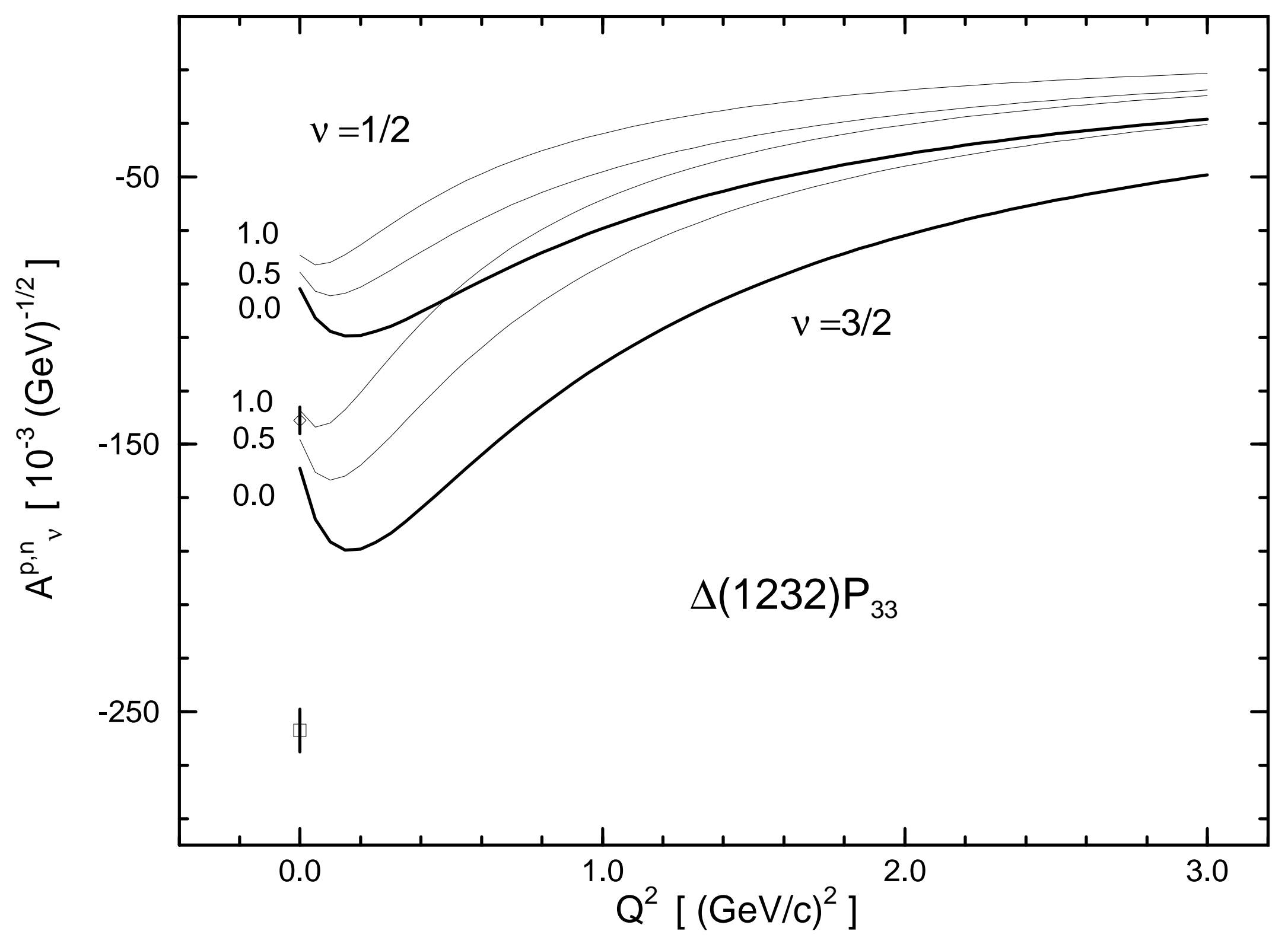




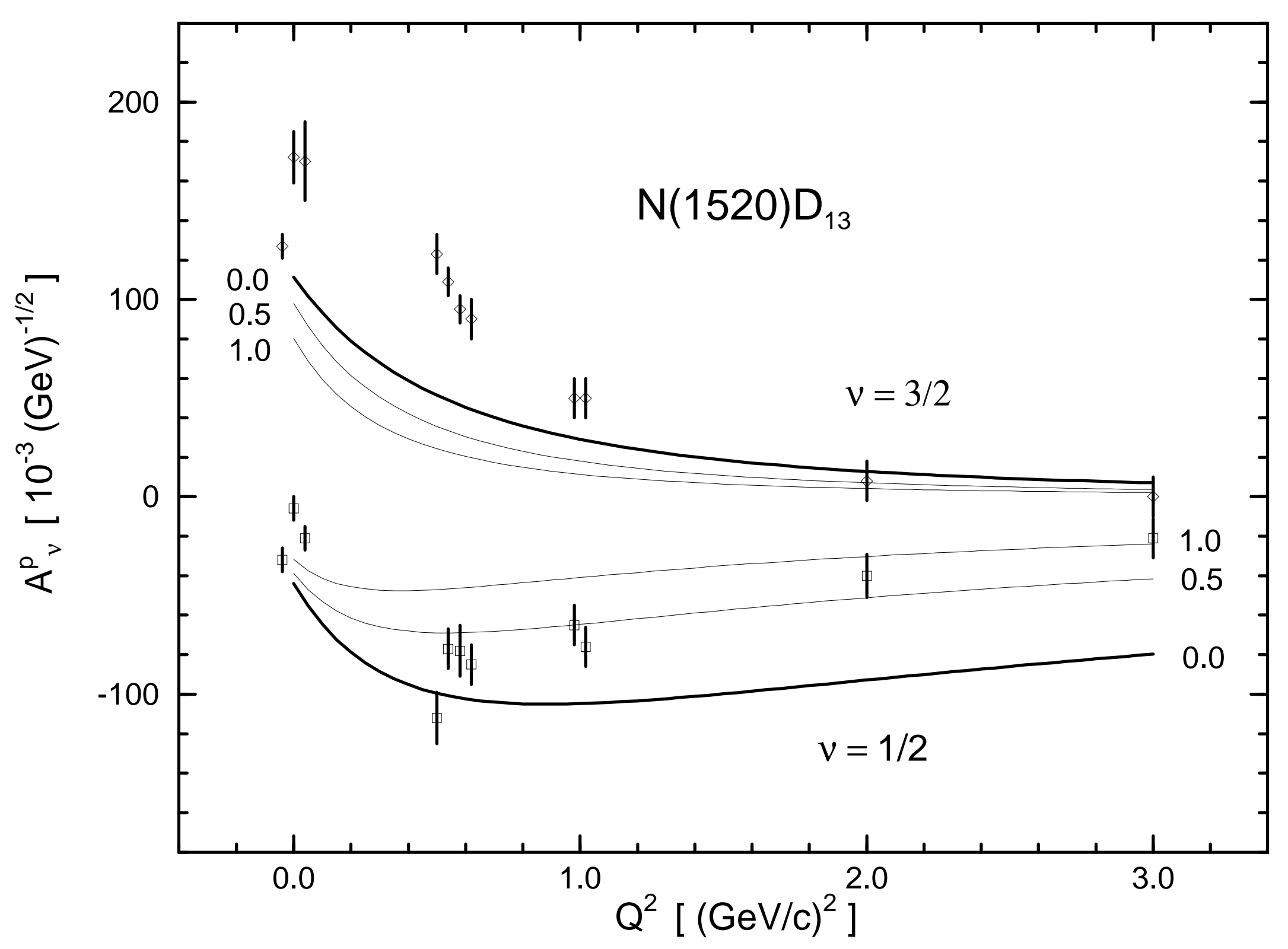




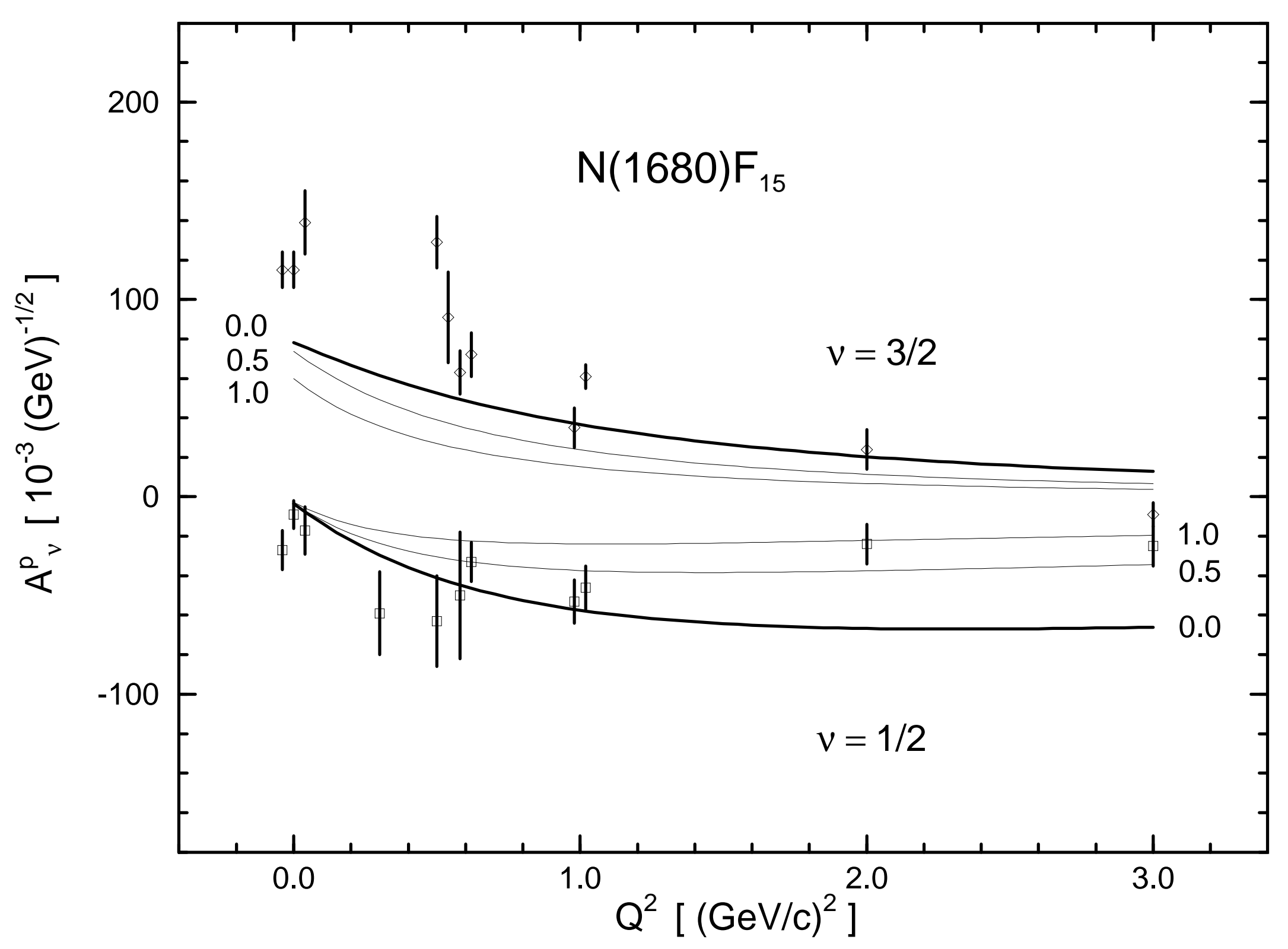

